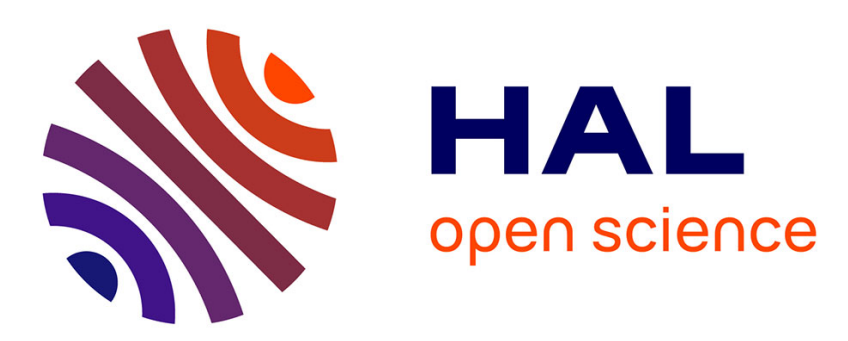

\title{
Characterization of the nonlinear behavior of nodular graphite cast iron via inverse identificationeAnalysis of uniaxial tests
}

Zvonimir Tomicevic, Janos Kodvanj, François Hild

\section{- To cite this version:}

Zvonimir Tomicevic, Janos Kodvanj, François Hild. Characterization of the nonlinear behavior of nodular graphite cast iron via inverse identificationeAnalysis of uniaxial tests. European Journal of Mechanics - A/Solids, 2016, 59, pp.140-154. 10.1016/j.euromechsol.2016.02.010 . hal-01303532

\section{HAL Id: hal-01303532 \\ https://hal.science/hal-01303532}

Submitted on 18 Apr 2016

HAL is a multi-disciplinary open access archive for the deposit and dissemination of scientific research documents, whether they are published or not. The documents may come from teaching and research institutions in France or abroad, or from public or private research centers.
L'archive ouverte pluridisciplinaire HAL, est destinée au dépôt et à la diffusion de documents scientifiques de niveau recherche, publiés ou non, émanant des établissements d'enseignement et de recherche français ou étrangers, des laboratoires publics ou privés. 


\title{
Characterization of the nonlinear behavior of nodular graphite cast iron via inverse identification
}

\author{
Analysis of uniaxial tests \\ Zvonimir Tomičevića,b, Janoš Kodvanj ${ }^{\mathrm{a}}$, François Hild ${ }^{\mathrm{b}}$ \\ ${ }^{a}$ Department of Engineering Mechanics, Faculty of Mechanical Engineering and Naval \\ Architecture, University of Zagreb, Ivana Lučića 5, 10000 Zagreb Croatia \\ ${ }^{b}$ LMT, ENS Cachan/CNRS/Université Paris-Saclay \\ 61 avenue du Président Wilson, 94235 Cachan Cedex, France
}

\begin{abstract}
The aim of this work is to estimate the parameters of elastoplastic and damage laws for nodular graphite cast iron from a cyclic uniaxial test on a dogbone sample. The paper focuses on the identification of material parameters coupling finite element models and full-filed measurements. The gap between the measured and simulated data is used to estimate the quality of the proposed constitutive postulates.

Last, a cyclic uniaxial experiment is carried out in a lab tomograph to reveal the damage micromechanism. Digital volume correlation is used to measure displacement fields in the bulk of the sample. The correlation residuals are used to detect the damage mechanism occurring in the heterogeneous microstructure of the material.

Keywords: Digital Image Correlation, Digital Volume Correlation, Full-field measurements, Material parameter identification, Nonlinear constitutive laws.
\end{abstract}




\section{Introduction}

The way of identifying material parameters to describe constitutive postulates has experienced major changes with the development of full-field measurement techniques (e.g., Digital Image Correlation (DIC), moiré and speckle interferometry, and grid methods [1]). Different approaches [2] are developed to obtain the sought parameters with various measured data (i.e., displacement or strain fields, load levels). These identification methods are applied to model different phenomena (e.g., elasticity [2, 3], plasticity $[4,5,6,7,8]$, damage $[9,10,11,12,13])$ from a wide range of experiments.

In a review on the identification of elastic properties [2] five different identification methods were introduced. In general the material parameters can be obtained by iteratively updating or direct identification procedures. The following iterative methods Finite Element Model Updating (FEMU), Constitutive Equation Gap Method (CEGM), Reciprocity Gap Method (RGM) use computed displacement and/or stress fields by FE analyses. An FE computation with an initial guess of the constitutive parameters is needed, while material parameters are identified by minimizing a cost function where the available measurements are compared with their computed counterparts. The cost functions for FEMU-U (displacement method), FEMU-F (force method) and FEMU-UF (displacement \& force method) are usually defined as quadratic errors of measured and computed values.

Contrary to updating procedures, non iterative methods such as the Virtual Fields Method (VFM) [14] and Equilibrium Gap Method (EGM) [9] use measured strain/displacement fields to determine material parameters [2]. In comparison with updating techniques a big advantage of these methods is 
the smaller duration of computation since they do not require a series of $\mathrm{FE}$ calculations.

Very few identifications have been conducted to account for elastoplasticity and damage. However, both phenomena were investigated separately with non-iterative (i.e., EGM [15, 16]), and iterative identification methods, namely, FEMU [5, 6, 8], VFM [17, 18], CEGM [19]. Damage identification from full-field measurements was first proposed by resorting to the equilibrium gap method [15]. It consists of a finite element formulation in which the nodal displacements are known and the elastic properties (i.e., the damage field) are sought. Elastic properties are assumed to remain uniform over each element, but vary from element to element. The damage field is nothing but a simple way to account for a heterogeneous stiffness within the sample. The additional step that has been tested against experimental data is to require for constitutive consistency, namely, that the damage inhomogeneity results from a homogeneous damage law combined with a heterogeneous loading [23]. EGM has the advantage of being directly applicable as a post-processor to the current displacement field measurement techniques such as digital image correlation.

Rossi et al. [24] identified damage parameters of Lemaitre's model from a uniaxial experiment. The main aim was to show how digital image processing may be used in material testing and characterization. DIC was used to obtain the plastic strain from which five parameters were obtained. Pierron et al. [18] made an extension of the virtual fields method to combine isotropic and kinematic hardening. Displacements were obtained by using the grid method. Since stress-related parameters were identified load measurements 
were included in the identification procedure. Based on the effect of noisy data to the cost function the choice of optimal virtual fields was investigated.

CEG-based methods [19] have also been proposed and applied in the context of nonlinear constitutive models. The identification of heterogeneous elastoplastic properties (i.e., linear kinematic hardening) and strain energy densities from kinematic field measurements have been addressed. The identification was based on an incremental version of CEGM where tangent or secant stiffness operators associated with elastoplastic models are sought.

Integrated DIC (or I-DIC) was also considered to tune elastoplastic [7, 8] and damage models [11] independently. Initially such types of techniques used closed-form (i.e., elastic) solutions [20, 21]. They were subsequently generalized by numerically generating sensitivity fields to material parameters for linear or nonlinear constitutive laws [22, 11, 8]. Very recently, it was shown that when properly weighted, FEMU and I-DIC lead to similar parameter uncertainties in the limit of small measurement uncertainties [8].

In the present paper weighted FEMU coupled with global DIC [27] will be used to tune elastoplastic material properties from a quasi-static uniaxial experiment. The proposed method was already applied for the identification of elastoplastic material parameters $[5,6,8]$. However, the cost functions were not weighted by covariance matrices associated with measurement uncertainties, which probe the level of each considered quantity with respect to its uncertainty level and accounts for correlations when needed. Three different constitutive postulates will be used herein to describe the nonlinear behavior (i.e., isotropic hardening, kinematic hardening and isotropic hardening coupled with damage). The main goal of the present paper is 
to determine the elastoplastic behavior coupled with damage by analyzing loading and unloading steps in one identification procedure. Second, the results obtained with different material models will be compared in terms of displacement and force residuals in order to provide the most reliable constitutive model among the proposed laws. Last, in order to detect damage micromechanisms an in-situ cyclic uniaxial test was performed in an X-ray tomograph. Regularized Digital Volume Correlation (RC8-DVC [40]) measures displacement fields in the observed volume. The correlation residuals will be used to detect damage.

\section{Mechanical test and material}

The material considered in this study is spheroidal graphite (SG) cast iron. At the macroscopic level SG cast iron is considered to be homogeneous with isotropic elastic properties although it is heterogeneous at a microscopic level. The heterogeneous microstructures of ductile cast iron consist of a ferritic matrix containing randomly distributed graphite inclusions (Figure 1).

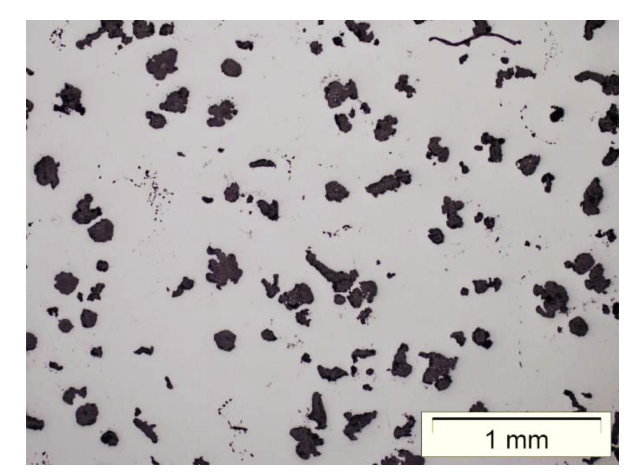

Figure 1: Metallography of the investigated SG cast iron 
A cyclic tensile test was carried out with an electro-mechanical testing machine on a dog-bone sample. The region of interest is approximately 50$\mathrm{mm}$ long, its ligament is $10-\mathrm{mm}$ wide, and the sample is 5 -mm thick. The measurement protocol used herein consisted of an optical setup (for DIC measurements) on one side of the sample while for validation purposes a strain gauge was mounted on the other side. Before the experiment, the specimen was prepared for DIC purposes. An artificial texture (i.e., speckle pattern) was applied with white and black paints sprayed over the ligament. The latter was observed with a single camera (i.e., 2D-DIC measurements). A telecentric lens with a magnification $\times 4$ was mounted on a PCOedge camera (16-bit digitization, definition: $2560 \times 2160$ pixels). The chosen lens allows artifacts related to out-of-plane motions to be minimized (if not completely canceled out). The physical size of one pixel on the acquired images corresponds to $52 \mu \mathrm{m}$. The strain gauge data were compared with DIC measurements to validate the latter ones with the present optical setup. A very good consistency was observed [35].

Figure 2 shows the cyclic loading path. The experiment was conducted in a displacement controlled mode with a loading rate of $3 \mu \mathrm{m} / \mathrm{s}$ except for the first cycle $(0.5 \mu \mathrm{m} / \mathrm{s})$. The first two cycles with maximum loads 8 and $12 \mathrm{kN}$ were performed in the elastic regime. After the third one (i.e., $15 \mathrm{kN}$ peak load) plastic strains were observed. Cycling was continued with 1-kN load increments in each following cycle. The camera was triggered with a testing machine output analog signal so that the load level is known for each acquired picture. After eleven unloading/reloading sequences the sample broke and 5,520 images were shot with an acquisition rate of 1 frame per second. The 
displacement fields were measured with a global T3-DIC code [46]. The correlation was performed with the reference image shot in the unloaded state and the deformed ones in the loaded state.

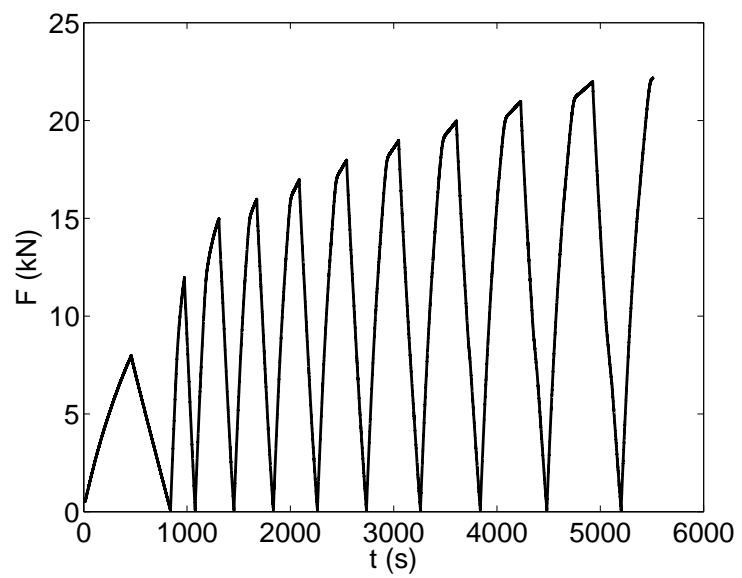

Figure 2: Loading history for the uniaxial cyclic experiment. The image acquisition rate was 1 frame/s

\section{Identification strategy}

\subsection{DIC measurement}

The general concept of DIC is pattern matching to measure the displacement field by correlating the image taken in the reference configuration $f(\mathbf{x})$ and other ones in the deformed configuration $g(\mathbf{x})$. Images $f$ and $g$ are matrices of gray level values where $\mathbf{x}$ denotes the position of pixels. For the two images gray level conservation (i.e., rule of optical flow) is usually assumed

$$
f(\mathbf{x})=g(\mathbf{x}+\mathbf{u}(\mathbf{x}))
$$

where $\mathbf{u}$ is the unknown displacement field to be measured. Global DIC [25, 26,27 considers the whole region of interest (ROI) of an image for correlation 
purposes to find the sought displacement field minimizing the sum of squared differences $\Phi_{c}^{2}$

$$
\Phi_{c}^{2}=\int_{\mathrm{ROI}} \varphi_{c}^{2}(\mathbf{x}) \mathrm{d} \mathbf{x}
$$

where $\varphi_{c}(\mathbf{x})$ defines the field of correlation residuals

$$
\varphi_{c}(\mathbf{x})=|f(\mathbf{x})-g(\mathbf{x}+\mathbf{u}(\mathbf{x}))|
$$

The objective of global DIC is to reduce measurement uncertainties with the reduction of the number of unknowns by introducing known information (i.e., continuity of the displacement between the elements found in finite elements [28]). The sought displacement field is written as $\mathbf{u}(\mathbf{x})=\sum_{n} u_{n} \boldsymbol{\psi}_{n}(\mathbf{x})$. Different forms of shape functions can be chosen to analyze the displacement fields (e.g., 4 noded quadrangles (Q4-DIC [27, 28]), three-noded triangles (T3-DIC [22, 46])). In the following studies, 3-noded elements will be considered. They are the simplest in terms of displacement interpolations, even though they are seldom used, if ever, in the framework of global DIC.

\subsection{Weighted Finite Element Model Updating}

The aim of this work is to identify elastoplasticity coupled with damage phenomena. When applying the minimization to strain fields [5] the management of the kinematic boundary conditions is not necessarily straight forward. When using displacement fields it becomes easier. Simulations and measurements (with local DIC [29, 30, 31]) are usually carried out with different softwares. As a consequence the reference points from measured and calculated data are not located at the same position. With additional interpolations the two fields are then compared. This is adding extra interpolation 
errors and uncertainties. On the contrary, global DIC is based on FE formulations. Hence, coupling FE simulations with global DIC within FEMU is a natural tool to determine material parameters because the same mesh can be used $[3,8]$. The results will be compared on the nodes of the very same mesh, which excludes any subsequent interpolation operation.

To determine elastic contrasts FEMU-U is appropriate [3]. However, if the sought mechanical parameters are affected by stresses the minimization of the cost function based on displacement/strain fields is not sufficient. This case requires the introduction of the global equilibrium gap in the identification procedure $[6,8]$. The identification is then the result of minimizing the total residual summed over all states where an image is captured [8]

$$
\chi_{t o t}^{2}=(1-\omega) \chi_{u}^{2}+\omega \chi_{F}^{2}
$$

where $\chi_{u}$ is the displacement residual, $\chi_{F}$ the force residual, and $\omega$ a (dimensionless) weight with limits $0 \leq \omega \leq 1$. Since different types of data are gathered in $\chi_{\text {tot }}$ it may be proposed to use $\omega=1 / 2$ so that the credit given to static and kinematic information is identical [8]. Depending on the value of $\omega$, different techniques are obtained:

- FEMU-U $(\omega=0)$

As mentioned earlier FEMU-U consists of minimizing the cost function as the squared norm error between measured $\left\{\mathbf{u}_{\mathbf{m}}\right\}$ (i.e., a vector gathering all measured nodal displacements) and computed $\left\{\mathbf{u}_{\mathbf{c}}\right\}$ (i.e., vector gathering all calculated nodal displacements) [3, 8]

$$
\chi_{u}^{2}(\{\mathbf{p}\})=\frac{1}{\gamma_{u}^{2} N_{u}}\left\|\left\{\mathbf{u}_{\mathbf{m}}\right\}-\left\{\mathbf{u}_{\mathbf{c}}\right\}\right\|^{2}
$$


where $\{\mathbf{p}\}$ is the column vector gathering all unknown material parameters of a chosen constitutive model, and $\gamma_{u}$ is the standard displacement uncertainty (if the measurements are uncorrelated). Since different data are considered in $\chi_{\text {tot }}$ (displacement and force residuals) it is necessary to introduce the normalization factor $1 / \gamma_{u}^{2} N_{u}$ [8], where $N_{u}$ denotes the total number of kinematic degrees of freedom. In the present case, the minimization is performed by resorting to the $\chi$-squared estimate

$$
\chi_{u}^{2}(\{\mathbf{p}\})=\frac{1}{N_{u}}\left(\left\{\mathbf{u}_{\mathbf{m}}\right\}-\left\{\mathbf{u}_{\mathbf{c}}\right\}\right)^{t}\left[\mathbf{C}_{\mathbf{u}}\right]^{-1}\left(\left\{\mathbf{u}_{\mathbf{m}}\right\}-\left\{\mathbf{u}_{\mathbf{c}}\right\}\right)
$$

where $\left[\mathbf{C}_{\mathbf{u}}\right]$ is the covariance matrix equal to $2 \gamma_{f}^{2}[\mathbf{M}]^{-1}[27]$, and $[\mathbf{M}]$ the global DIC matrix.

The minimization of $\chi_{u}^{2}$ is performed by successive linearizations and corrections. The new estimate (i.e., at iteration $i$ ) of the computed displacement field reads

$$
\mathbf{u}_{\mathbf{c}}\left(\mathbf{x}, t,\left\{\mathbf{p}^{(i)}\right\}\right)=\mathbf{u}_{\mathbf{c}}\left(\mathbf{x}, t,\left\{\mathbf{p}^{(i-1)}\right\}\right)+\frac{\partial \mathbf{u}_{\mathbf{c}}}{\partial\{\mathbf{p}\}}\left(\mathbf{x}, t,\left\{\mathbf{p}^{(i-1)}\right\}\right)\{\delta \mathbf{p}\}
$$

where $\left\{\mathbf{p}^{(i-1)}\right\}$ is the set of parameters at iteration $i-1$, and $\{\delta \mathbf{p}\}$ the sought parameter increment. The computation of the sensitivity fields

$$
[\mathbf{S}]=\frac{\partial \mathbf{u}_{\mathbf{c}}}{\partial\{\mathbf{p}\}}
$$

is performed numerically by computing displacement fields for small variations of each parameter of the considered set. For iteration $i$ it is necessary to calculate the sensitivity fields and once they are obtained the correction of the sought parameters read

$$
\{\delta \mathbf{p}\}=\left[\mathbf{H}^{(i-1)}\right]^{-1}\left[\mathbf{S}^{(i-1)}\right]^{t}[\mathbf{M}]\left(\left\{\mathbf{u}_{\mathbf{m}}\right\}-\left\{\mathbf{u}_{\mathbf{c}}{ }^{(i-1)}\right\}\right)
$$


where $\left[\mathbf{H}^{(i-1)}\right]=\left(\left[\mathbf{S}^{(i-1)}\right]^{t}[\mathbf{M}]\left[\mathbf{S}^{(i-1)}\right]\right)^{-1}$ is the approximate Hessian. Convergence is usually written in terms of relative variations of the sought parameters.

- $F E M U-F(\omega=1)$

Similar to FEMU-U, the $\chi$-squared equilibrium gap $\chi_{F}$ is minimized

$$
\chi_{F}^{2}(\{\mathbf{p}\})=\frac{1}{\gamma_{F}^{2} N_{F}}\left\{\mathbf{F}_{\mathbf{c}}-\mathbf{F}_{\mathbf{m}}\right\}^{t}\left\{\mathbf{F}_{\mathbf{c}}-\mathbf{F}_{\mathbf{m}}\right\}
$$

where $\gamma_{F}$ is the standard resolution of the load measurement, and $N_{F}$ the total number of load data. Thus the expected value of $\chi_{F}$ is unity when only noise is at play. The identification is then the result of the minimization of $\chi_{F}^{2}$.

- FEMU-UF $(0<\omega<1)$

The last procedure is a combination of the two previous approaches [8]. To treat all measured data on the same footing,

$$
\omega=\frac{N_{F}}{N_{F}+N_{u}} .
$$

Under such conditions the total cost function is consistent with a Bayesian framework. This weighting will be used for all the results reported hereafter.

\subsection{Implementation}

As previously presented, FEMU methods rely on the comparison of the measured displacement fields and reaction forces with those calculated by FE 
simulations. It is mandatory for the calculated and the measured displacements to be compared at the same locations. In the present case, the measurement of the displacement field is carried out with the same mesh as that used in simulations. The dialog between simulation and experimental data can also be limited by the common assumption that the loading conditions of the simulation are associated with ideal boundary conditions that are not reflecting the inherent imperfections of the test. On the FE model boundary conditions extracted from the experiment can also be applied (e.g., measured displacements, static loading information).

In this work the identification is carried out on a simple specimen geometry. Hence, a 2D model in the commercial FE code Abaqus is created from the T3-DIC mesh. Dirichlet boundary conditions prescribed in the simulations will be extracted from DIC measurements (Figure 3). It is important to

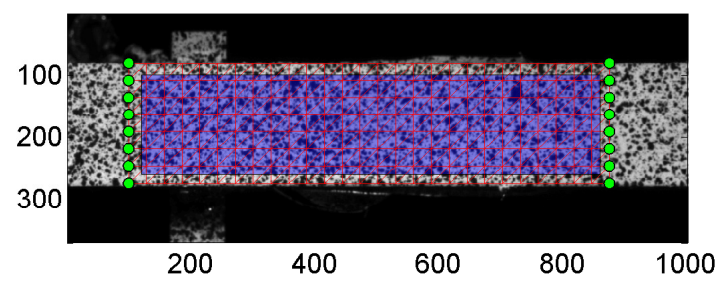

Figure 3: Reference picture of the uniaxial experiment used for a DIC analysis with 25pixel elements. The blue rectangle depicts the zone where the DIC gauge was measuring the mean strain. In the FEMU procedure, boundary conditions measured with DIC were prescribed on the edge nodes depicted with green circles

emphasize that many issues can influence the performance of an experiment. Usually, even simple tests (e.g., uniaxial tension) are not perfect and their response includes some anomalies. Observing the displacement field in the 
transverse direction (Figure 4(a)) an additional rotation is noted even though the test was carried out in tension. Applying the measured displacements to the boundary of the FE model accounts for these imperfections. The material parameters obtained by curve fitting of a monotonic tensile test in which the mean strain was measured via DIC analyses and coupled with the load data will be used as initial inputs for the FEMU procedure. Displacement fields and reaction forces are calculated in Abaqus and extracted via Phyton scripts. The FEMU-UF procedure is carried out in Matlab to minimize the total chi-squared error (Equation (4)).

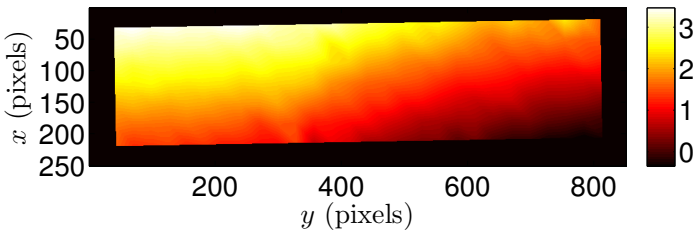

(a)

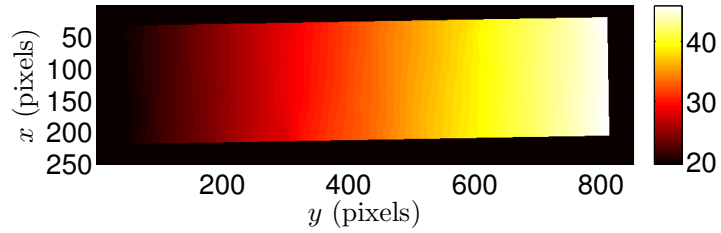

(b)

Figure 4: Measured displacement fields via T3-DIC in (a) transverse and (b) longitudinal directions for a load level corresponding to $18 \mathrm{kN}$. The displacements are expressed in pixels $(1$ pixel $\leftrightarrow 52 \mu \mathrm{m})$

\section{Nonlinear constitutive models}

The first three postulates to be calibrated herein are built-in models while the damage model has been implemented via a User Material (UMAT) subroutine. The following postulates are selected:

- Linear isotropic elasticity

Before identifying the nonlinear material parameters it was necessary to 
tune the elastic parameters (i.e., Poisson' ratio $\nu$ and Young's modulus $E)$.

- Ludwik's isotropic hardening law

Ludwik's law [32] is considered for describing isotropic hardening. Its general expression is

$$
\sigma_{e q}=\sigma_{y}+K p^{n}
$$

where the sought parameters are the yield stress $\sigma_{y}$, the hardening modulus $K$ and the hardening exponent $n . \sigma_{e q}=\sqrt{\frac{2}{3} \sigma_{i j}^{D} \sigma_{i j}^{D}}$ corresponds to von Mises' equivalent stress, $\sigma_{i j}^{D}$ the deviatoric stress tensor, and $p$ the cumulated plastic strain.

- Armstrong-Frederick's kinematic hardening

Armstrong and Frederick's model [33] is an upgraded version of Prager's linear kinematic law [34] in which a memory term is added

$$
\dot{\mathbf{X}}=C \dot{\varepsilon}_{p l}+\gamma \mathbf{X} \dot{p}
$$

where $\mathbf{X}$ is the back-stress tensor, $C$ and $\gamma$ are material parameters to be identified and $\dot{p}$ the rate of cumulated plastic strain. When compared with experimental results, Armstrong-Frederick's predictions are generally more accurate than Prager and von Mises's models for cyclic uniaxial loadings and torsion-tension of thin tube tests [33].

- Lemaitre's damage model

The derivation of Lemaitre's damage law [36] unifies a damage model coupled with Ludwik's elastoplastic hardening (Equation (12)). The 
damage growth is written as

$$
\dot{D}=\left(\frac{Y}{S}\right)^{s} \dot{p} \quad \text { when } \quad p \geq p_{t h}
$$

where $s$ and $S$ are material-dependent parameters, and $p_{t h}$ the strain equivalent strain below which damage does not grow. The identification will consist of determining those two parameters while in the same procedure the corresponding Ludwik's parameters describing the elastoplastic behavior will be sought. The energy release rate density $Y$ is the associated force with the damage variable

$$
Y=\frac{\tilde{\sigma}_{e q}^{2} R_{\nu}}{2 E}
$$

where $R_{\nu}$ is the triaxiality function

$$
R_{\nu}=\frac{2}{3}(1+\nu)+3(1-2 \nu)\left(\frac{\sigma_{H}}{\sigma_{e q}}\right)^{2}
$$

and $\sigma_{H}=\sigma_{k k} / 3$ the hydrostatic stress, $\widetilde{\sigma}_{e q}=\sigma_{e q} /(1-D)$.

\section{FEMU results}

\subsection{Elastic parameters}

The aim of the present subsection is to identify Young's modulus and Poisson's ratio in a single identification procedure. The FEMU-UF variant is chosen. Before performing the identification the measurement uncertainty was estimated on 10 images acquired when the specimen was mounted and without loading. The standard displacement resolution is equal to 0.004 pixel and the corresponding load resolution $\gamma_{F}$ is equal to $5 \mathrm{~N}$.

The identification was performed on the first 929 images that correspond to the first two loading cycles. The initial parameters for the FEMU-UF 
analysis are given in Table 1. The reference value for the Young's modulus was taken from a standard curve fitting on the linear part of the monotonic curve. The initial value of Poisson's ratio was chosen to be equal to 0.33. The identified Young's modulus via FEMU-UF (Table 1) changes slightly in

Table 1: Initial and identified elastic parameters for cyclic uniaxial tension

\begin{tabular}{ccccc}
\hline Parameter & $\mathrm{E}$ & $\nu$ & $\chi_{u}$ & $\chi_{F}$ \\
& $(\mathrm{GPa})$ & & & \\
\hline Reference $^{\natural}$ & 156 & 0.33 & 2.22 & 60 \\
FEMU-UF & $158 \pm 1$ & $0.28 \pm 0.00$ & 2.19 & 50 \\
\hline
\end{tabular}

घ: identified from a monotonic test [35]

comparison with the initial value. This is mainly causing a decrease of $\chi_{F}$ while a small decrease of $\chi_{u}$ is induced by the identified Poisson's ratio, which corresponds to the value measured with the strain gauge data [35]. The fact that the force residual is still high is presumably due to the fact that measured kinematic boundary conditions induce an additional contribution [37], which is neglected in the present case.

It is worth noting that the identified parameters have their uncertainties evaluated by propagating the displacement and load uncertainties to the material parameters [8]. Consequently, the identified parameters are reported with the corresponding \pm standard uncertainty due to the previously mentioned uncertainties. In the present case, the uncertainties associated with both elastic parameters are very small thanks to the coupling of kinematic and static data in the minimization procedure. 


\subsection{Ludwik's parameters}

The identification of Ludwik's parameters was performed on all acquired images (i.e., 5,520 pictures). The first calculation of the FEMU-UF analysis yielded high levels of normalized force residuals (i.e., $\chi_{F}=350$ ) in comparison with the elastic case (Table 1). The reference plastic parameters (i.e., obtained from a monotonic test [35]) do not describe in a reliable way the cyclic loading history (Figure 5), which is to be expected from an isotropic hardening model.

The identified values of the sought parameters $\left(\sigma_{y}, K\right.$ and $\left.n\right)$ given in Table 2 decrease $\chi_{u}$ and $\chi_{F}$. Figure 5 shows the comparison of the measured force and calculated sum of reaction forces for both sets of parameters. It is observed that the yield stress decreases by almost $30 \%$, which requires parameters $K$ and $n$ to change as well. The uncertainties on the identified parameters remain very small and of the same order of magnitude as what was observed for the elastic parameters.

For comparison purposes the elastic (see Table 1) and elastoplastic analyses can be studied. When the whole experiment (i.e., 5,520 images) is analyzed $\chi_{u}=37$ while for the elastic identification (950 images) $\chi_{u}=2.2$. The fact that lower levels of $\chi_{u}$ are obtained when compared with the elastoplastic analysis indicates a model error. The same trend is observed for the load residuals.

Figure 5 shows that higher levels of $\chi_{F}$ are due to a model error. The stiffness of the specimen is too high after the second cycle since the sum of reaction forces does not stop at $0 \mathrm{~N}$ upon unloading. This leads to the hypothesis that damage may occur very early on. Before discussing this 
Table 2: Identified Ludwik's parameters via FEMU-UF

\begin{tabular}{cccccc}
\hline Parameter & $\begin{array}{c}\sigma_{y} \\
(\mathrm{MPa})\end{array}$ & $\begin{array}{c}K \\
(\mathrm{MPa})\end{array}$ & $n$ & $\chi_{u}$ & $\chi_{F}$ \\
\hline Reference $^{\natural}$ & 289 & 1260 & 0.62 & 40 & 350 \\
FEMU-UF $^{2}$ & $207 \pm 2$ & $1300 \pm 15$ & $0.44 \pm 0.005$ & 37 & 240 \\
\hline
\end{tabular}

घ: identified from a monotonic test [35]

phenomenon, let us first analyze the experiment with a kinematic hardening model.

\subsection{Armstrong-Frederick's parameters}

The identification of Armstrong-Frederick's nonlinear kinematic hardening law was performed over the whole cyclic experiment. The reference parameters were also taken from the monotonic test (Table 3). The sought Armstrong-Frederick parameters for the cyclic loading history take different values than those obtained in monotonic loading. Figure 6 illustrates the different load levels reached. The initial parameters better describe the unloading parts of the history, while the identified parameters better capture the various fluctuations of the loading path.

The parameters identified for a cyclic loading history yield a significantly lower value of $\chi_{F}$ with respect to the parameters describing the monotonic test. Further, when the same test is identified they lead to lower residuals than Ludwik's law (Table 2). The fact that the loading cycles are not fully captured may be due to damage. This hypothesis will be checked in the following section. The uncertainties on the identified parameters are still very small in the present case. 


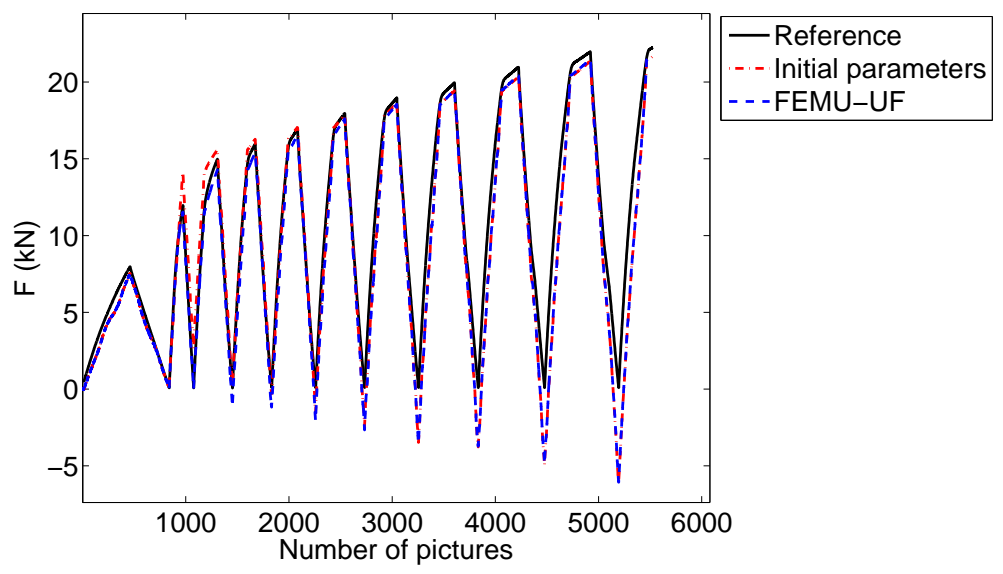

(a)

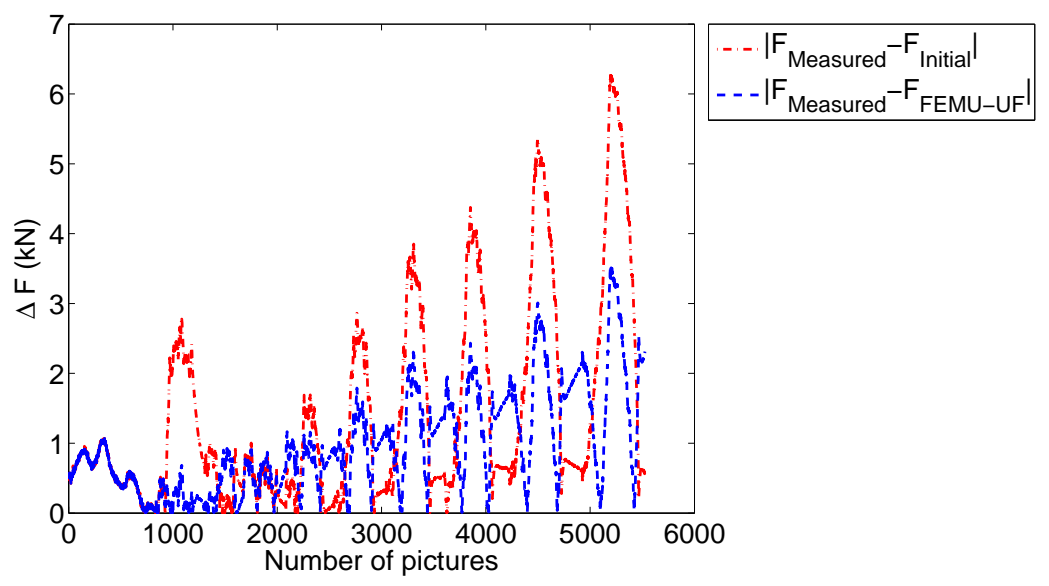

(b)

Figure 5: (a) Comparison of measured force and sum of reaction forces computed with initial and identified material parameters for Ludwik's law. (b) Difference between measured and predicted load levels for the initial and optimized elastic parameters 


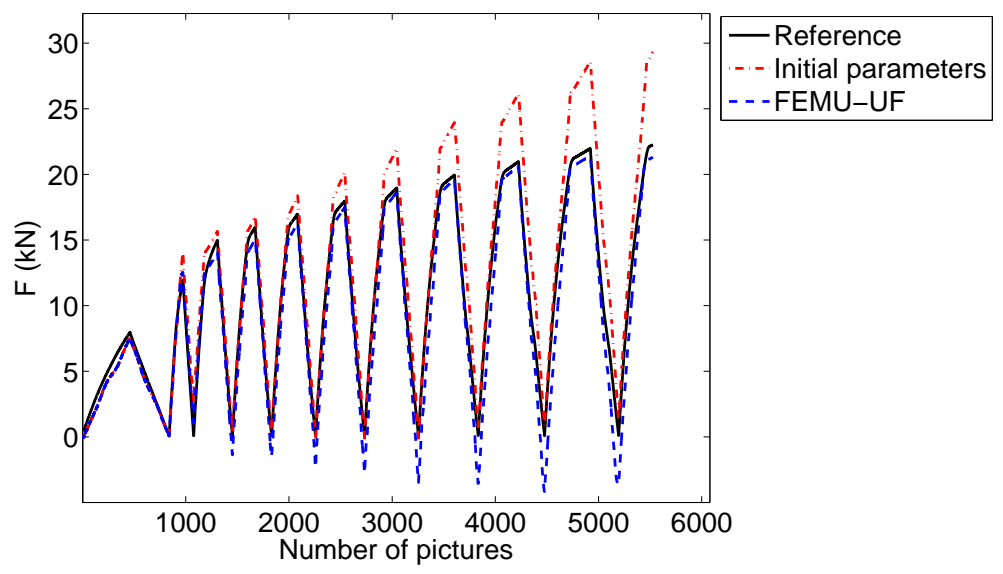

(a)

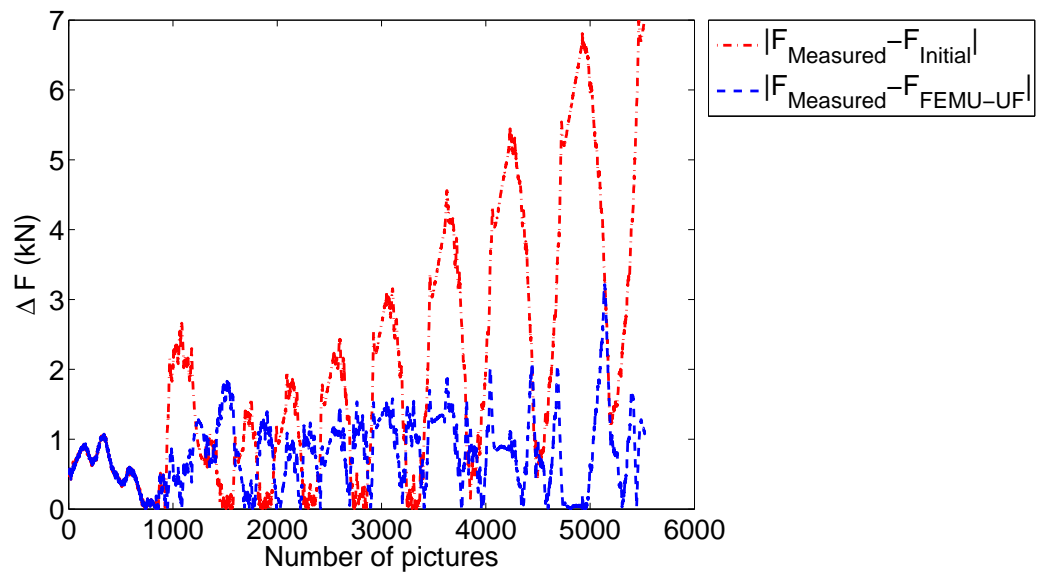

(b)

Figure 6: (a) Comparison of measured force and sum of reaction forces computed with the initial and identified parameters for Armstrong-Frederick's constitutive law. (b) Difference between measured and predicted load levels for the initial and optimized parameters 
Table 3: Identified Armstrong-Frederick's parameters with the FEMU-UF variant

\begin{tabular}{cccccc}
\hline Parameter & $\begin{array}{c}\sigma_{y} \\
(\mathrm{MPa})\end{array}$ & $\begin{array}{c}C \\
(\mathrm{GPa})\end{array}$ & & $\chi_{u}$ & $\chi_{F}$ \\
\hline Reference $^{\natural}$ & 289 & 10 & 45 & 35 & 400 \\
FEMU-UF & $222 \pm 0.3$ & $27 \pm 0.1$ & $106 \pm 0.5$ & 36 & 180 \\
\hline
\end{tabular}

t: identified from monotonic test [35]

\subsection{Lemaitre's damage parameters}

Without including damage phenomena in the identification procedure of Ludwik's model it is not possible to describe the cyclic behavior in a reliable way. Since damage represents the progressive material deterioration, it can be defined in terms of relative reduction of the net section of a representative volume element (RVE). With the additional assumption of isotropic damage, the damage tensor is reduced to a scalar $D$ for which a practical definition is given by [36]

$$
D=1-\frac{\tilde{E}}{E_{0}}
$$

where $E_{0}$ and $\tilde{E}$ are Young's moduli of the undamaged and damaged material.

To identify the nonlinear behavior from a cyclic experiment isotropic hardening Ludwik's law is coupled with Lemaitre's damage model in a single UMAT routine. Since FE calculations considering damage are time consuming the identification will first be carried out on one element. The identified parameters will give closer initial guesses for the complete FE model. 


\subsubsection{Results on one element}

One cube element with unitary size is used to obtain a first evaluation of the damage parameters. Two boundary conditions are prescribed on the simple model. First, the bottom surface is motionless in the loading direction, while on the upper surface the displacement is prescribed in the vertical direction. The prescribed displacement corresponds to measured axial strains (Figure 7(a)) with the DIC gauge (Figure 3). The identification procedure consists of minimizing the load difference from the cyclic stress-strain data (Figure 7(b)), which corresponds to FEMU-F.

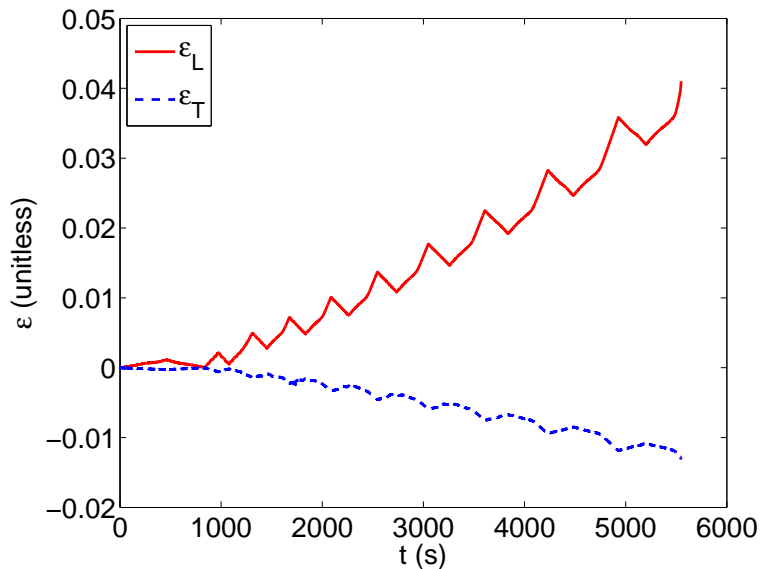

(a)

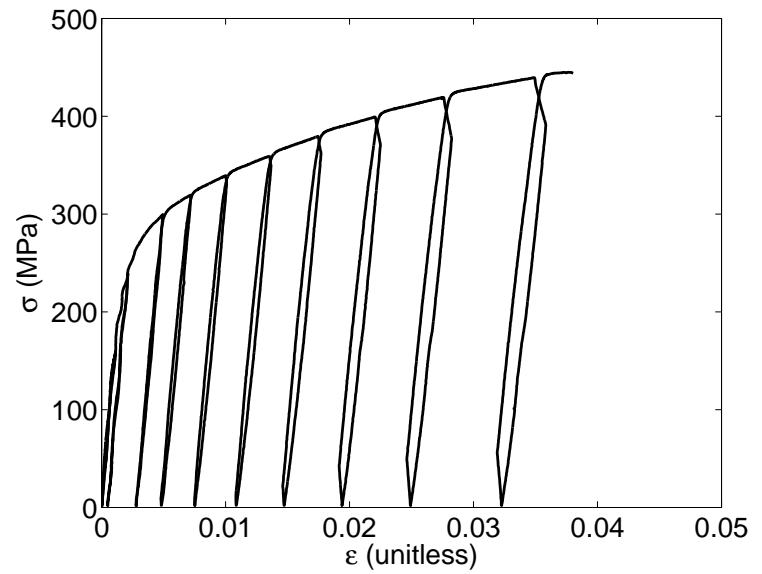

(b)

Figure 7: (a) Strain history in longitudinal (i.e., axial) and transverse directions. (b) Stress/strain curve for the cyclic uniaxial test

The sought parameters describing isotropic hardening are extended with the two damage parameters ( $S$ and $s$ ) of Lemaitre's law (Equation (14)). Except the two parameters it is necessary to define an equivalent plastic strain threshold (i.e., $p_{t h}$ at which damage starts to grow). To have access to the 
latter, the change of Young's modulus for each loading/unloading cycle was studied (Figure 8). An early loss of stiffness is detected. Hence, $p_{t h}$ is set to 0 . Apart from setting the plastic strain threshold, it is mandatory to provide a limit defining microcrack initiation (i.e., $D>D_{c r}$ ). Since $D_{c r}$ was difficult to identify, an arbitrary value of 0.3 was chosen from the literature [38].

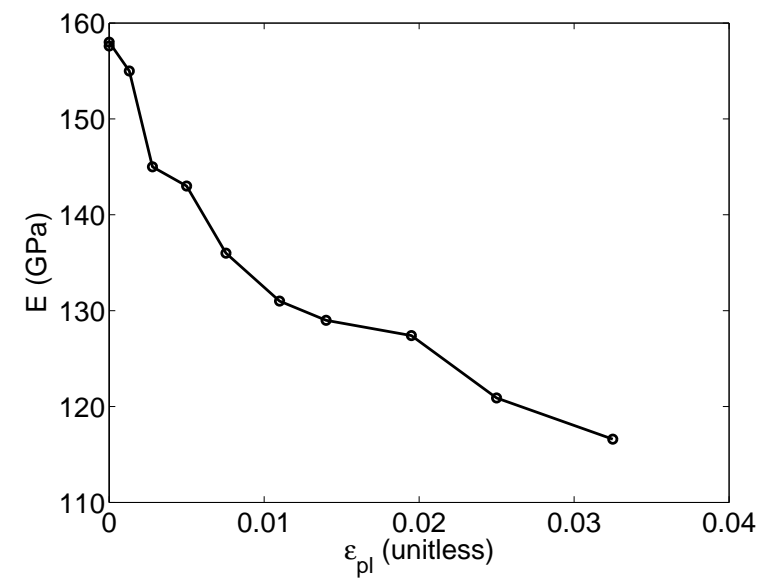

Figure 8: Change of Young's modulus with cumulative plastic strain

The FEMU-F procedure aims to identify/validate six parameters $\left(E, \sigma_{y}\right.$, $K, n, S$ and $s$ ). Initial values of the sought parameters are shown in Table 4. The reference elastoplastic parameters are chosen from the monotonic test while the damage parameters were arbitrarily chosen from the literature [38]. During the first few identification calculations it was noted that it is not possible to identify both damage parameters at the same time. Hence, the first iterations consisted of the identification of all the parameters except the damage exponent $s$, and the second one just focused on $s$. This approach successfully identified the sought parameters (see Table 4).

The biggest change of identified elastoplastic parameters was the hard- 
Table 4: Identified elastoplastic and damage parameters on one element via FEMU-F

\begin{tabular}{cccccccc}
\hline Parameter & $\begin{array}{c}E \\
(\mathrm{GPa})\end{array}$ & $\begin{array}{c}\sigma_{y} \\
(\mathrm{MPa})\end{array}$ & $\begin{array}{c}K \\
(\mathrm{MPa})\end{array}$ & & $\begin{array}{c}n \\
(\mathrm{MPa})\end{array}$ & & \\
\hline Reference $^{\natural}$ & 157.6 & 207 & 1257 & 0.62 & 0.5 & 1.5 & 540 \\
FEMU-F & $158.3 \pm 0.3$ & $242 \pm 1$ & $2880 \pm 39$ & $0.61 \pm 0.005$ & $0.25 \pm 0.02$ & $1.2 \pm 0.1$ & 180 \\
\hline
\end{tabular}

घ: $\left(E, \sigma_{y}, K, n\right)$ identified from monotonic test [35] and $(S, s)$ obtained from the literature [38]

ening modulus $(57 \%)$ and yield stress $(20 \%)$ while Young's modulus and hardening exponent hardly changed. The damage parameters also decreased ( $S$ by $50 \%$ and $s$ by $25 \%$ ). Figure 9 shows the comparison of the measured load levels and sum of reaction forces for the initial and identified parameters. The decrease of $\chi_{F}$ is observed in Table 4 , which results in a better agreement with the measured load levels obtained with the identified parameters.

\subsubsection{Results for FE model}

The same identification procedure as in the previous evaluation was applied to determine the sought parameters on the complete FE model of the test. The initial parameters are those obtained from the previous identification procedure (Table 5) and the identified results slightly differ from those obtained on one element. The uncertainties on the identified parameters are higher when compared to the results of Section 5.2 for the same hardening postulate. For the two damage parameters the coefficient of variation (i.e., the ratio of the standard uncertainty to the mean value) is one order of 


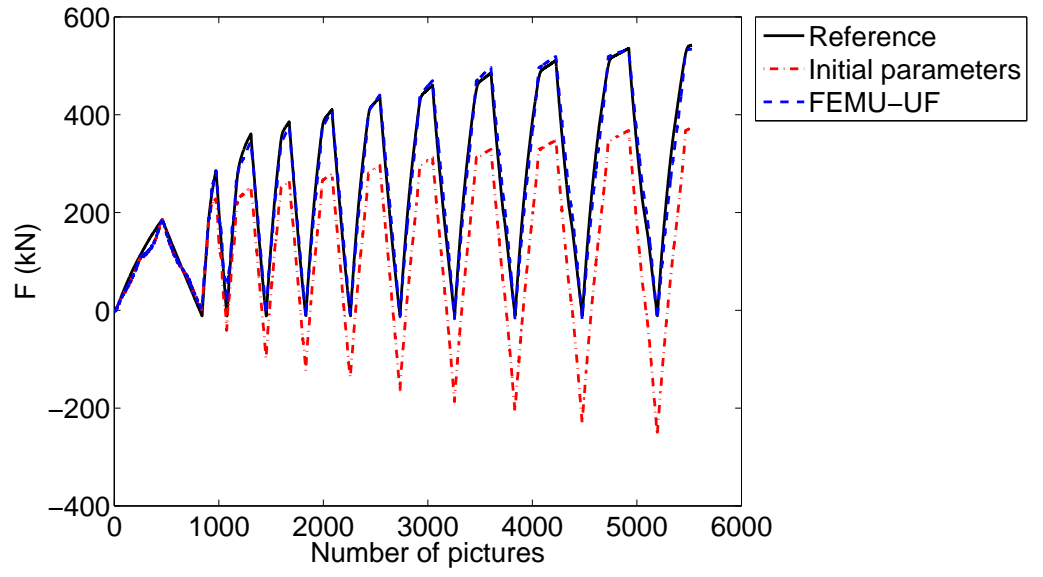

(a)

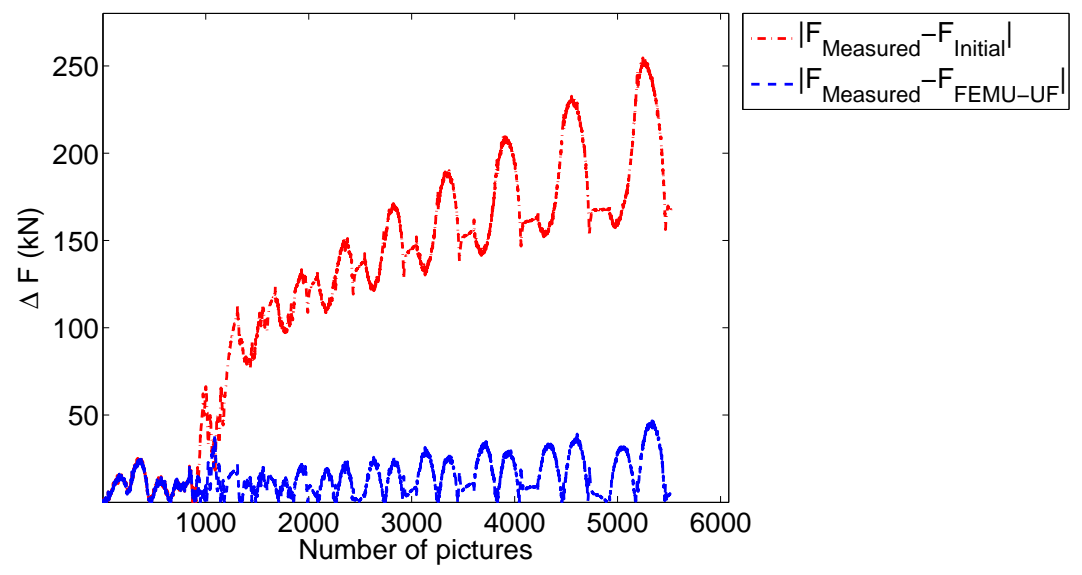

(b)

Figure 9: (a) Comparison of the measured load and sum of reaction forces computed with the initial and identified material parameters of an elastoplastic law coupled with damage for one element. (b) Difference between measured and predicted load levels for the initial and optimized parameters 
magnitude higher than that of the elastoplastic parameters, which is an additional proof that the identification of the damage parameters is very delicate.

Table 5: Identified elastoplastic and damage parameters with an FE model

\begin{tabular}{ccccccccc}
\hline Parameter & $\begin{array}{c}E \\
(\mathrm{GPa})\end{array}$ & $\begin{array}{c}\sigma_{y} \\
(\mathrm{MPa})\end{array}$ & $\begin{array}{c}K \\
(\mathrm{MPa})\end{array}$ & $n$ & $\begin{array}{c}S \\
(\mathrm{MPa})\end{array}$ & & $\chi_{u}$ & $\chi_{F}$ \\
\hline Reference $^{\natural}$ & 158.3 & 242 & 2880 & 0.61 & 0.25 & 1.2 & 35 & 150 \\
FEMU-UF & $157.9 \pm 0.4$ & $234 \pm 1$ & $2530 \pm 56$ & $0.59 \pm 0.005$ & $0.26 \pm 0.03$ & $1.2 \pm 0.1$ & 34 & 90 \\
\hline \multicolumn{7}{c}{ घ: analysis of Section 5.4 .1}
\end{tabular}

Figure 10 shows a good agreement between the sum of reaction forces extracted from the displacement prescribed boundaries with the measured load (in comparison with the previous case shown in Figure 5). Both components of the total residual decrease in comparison with the elastoplastic case (Table 2). Hence, the coupled elastoplastic and damage model describes in a better way the cyclic experiment. Similarly, the present identification out-performs that with kinematic hardening since the latter leads to load residuals 1.4 times higher than the former.

\subsection{Discussion}

Three different constitutive postulates have been tested herein. They could be ranked thanks to the weighted displacement and load residuals $\chi_{u}$ and $\chi_{F}$ (Table 6). The displacement residual remains high in comparison to the elastic case (Table 1). Conversely, the load residual for the damage 

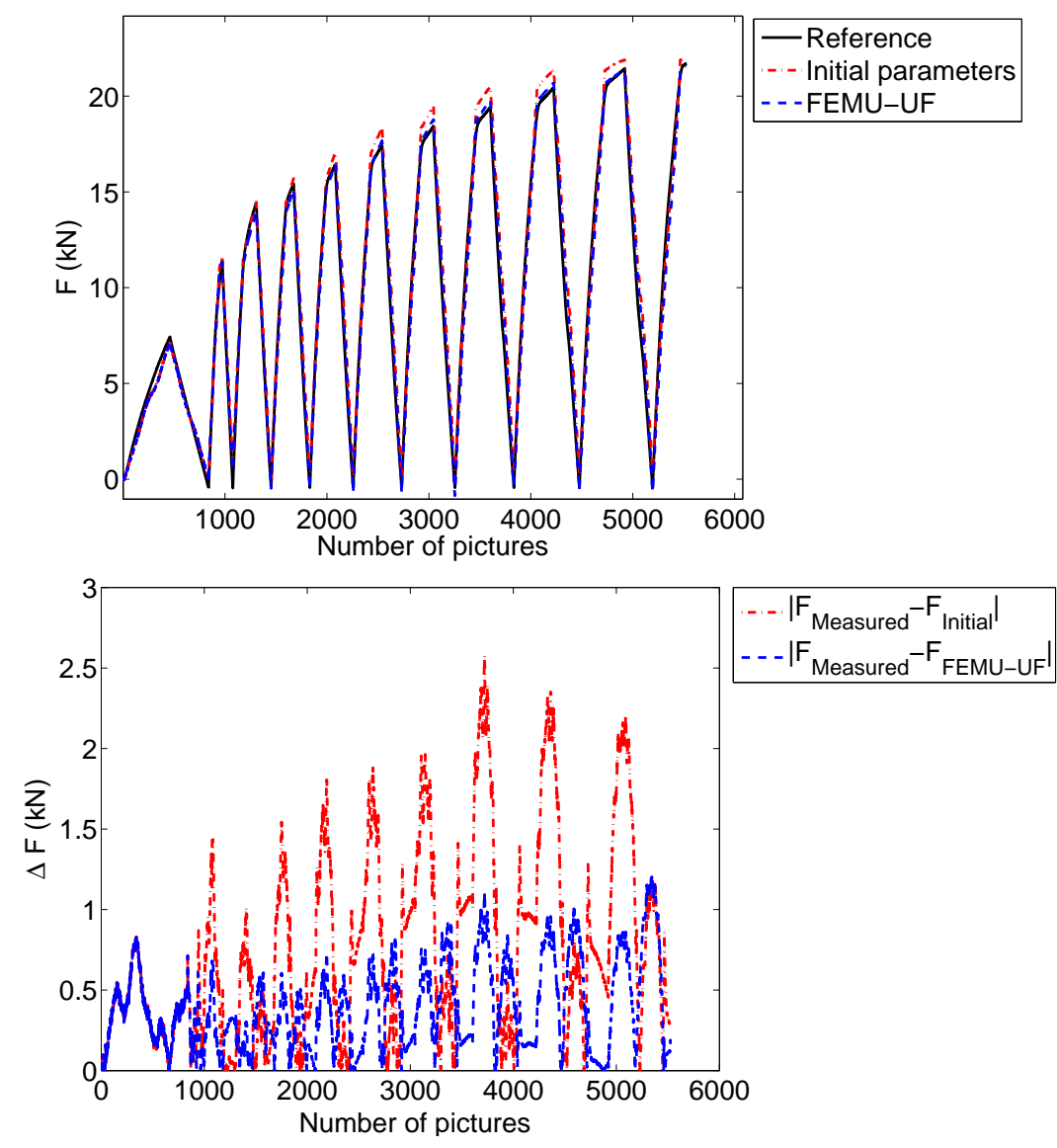

Figure 10: (a) Comparison of measured force and sum of reaction forces computed with the initial and identified parameters of the elastoplastic and damage law. (b) Difference between measured and predicted load levels for the initial and optimized parameters 
model is less than two times that obtained for an elastic case. This result further validates this model in comparison with the other two elastoplastic postulates.

Table 6: Identification residuals for the three analyzed models

\begin{tabular}{cccc}
\hline Model & Ludwik & Armstrong \& Frederick & Lemaitre \\
\hline$\chi_{u}$ & 37 & 36 & 34 \\
$\chi_{F}$ & 240 & 180 & 90 \\
$\chi_{\sigma}$ & 230 & 180 & 130 \\
\hline
\end{tabular}

The comparison can be further extended by using the longitudinal strain measurements and the mean longitudinal stress inferred from the load measurements. These data constitute the reference that is compared with the prediction of the three models when applied to the same volume element as in Section 5.4.1. Figure 11(a) shows the corresponding stress strain curves. From the stress differences shown in Figure 11(b) it can be concluded that the best prediction is obtained with the damage model coupled with isotropic hardening.

In quantitative terms, the RMS stress difference when normalized by the corresponding standard stress resolution defines $\chi_{\sigma}$. Table 6 shows that the order of magnitude of $\chi_{\sigma}$ and $\chi_{F}$ are similar. However, for the damage model, the FEMU-UF procedure leads to static residuals that are notably lower than those that would be obtained by a procedure assuming that the test is pure tension. 


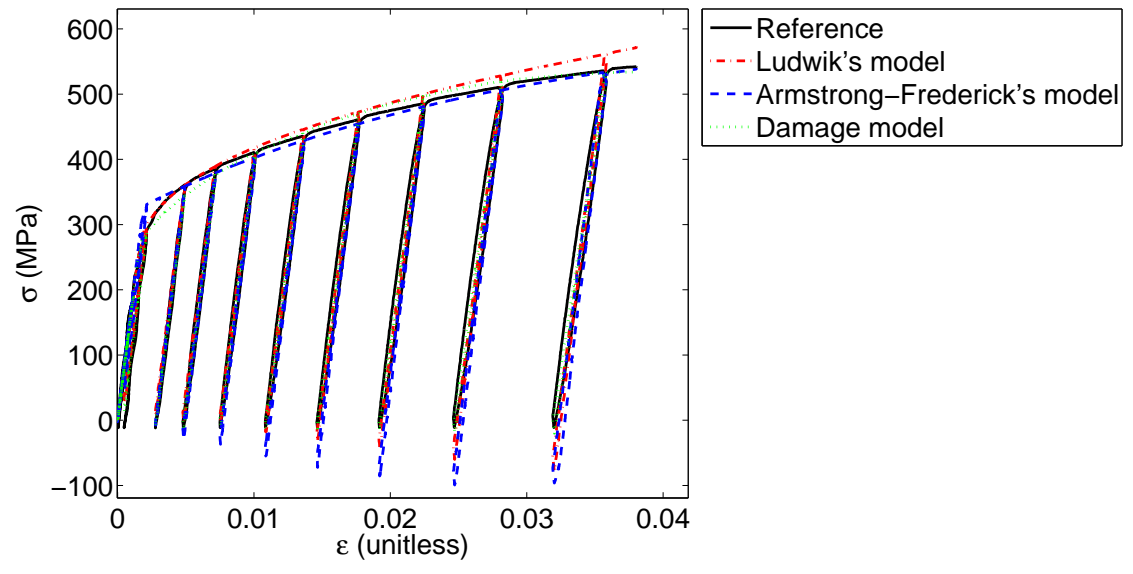

(a)

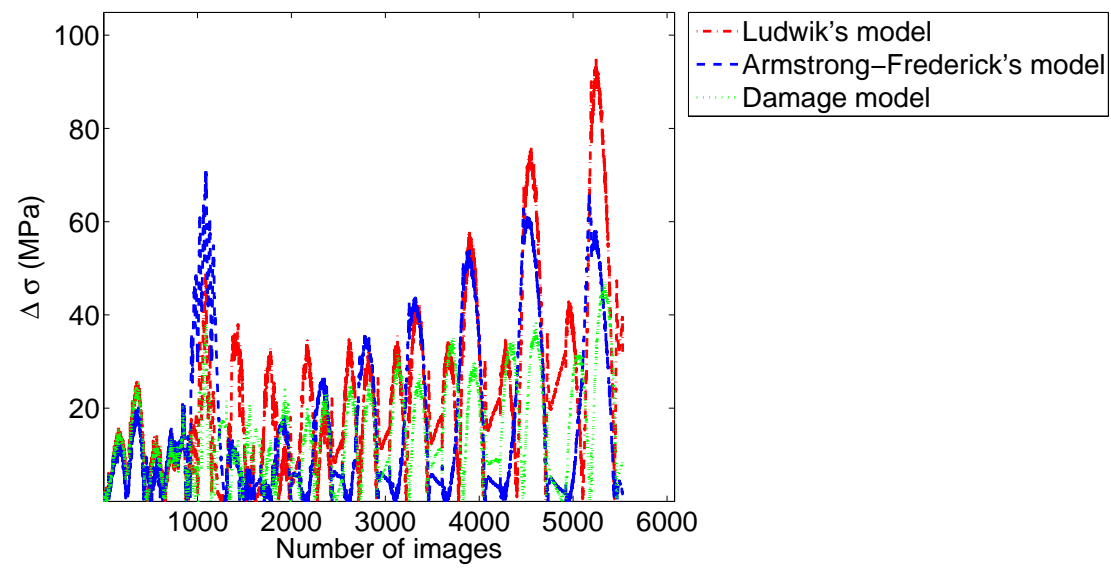

(b)

Figure 11: Comparison between the uniaxial stress/strain response of the three identified models and the reference obtained with strain gauge and load cell data. (b) Difference between measured and predicted stresses by the three studied models 


\section{Detection of damage micromechanisms via X-ray tomography}

In the previous sections cast iron was studied at the macroscopic level without any access to phenomena appearing within its heterogeneous microstructure. In this section a cyclic uniaxial experiment carried out in an

$\mathrm{X}$-ray tomograph is presented. The aim is to analyze from the displacement and residual fields given by volumetric correlation the interaction of the ferritic matrix and the graphite nodules under tensile loading. In particular, the development of damage will be discussed.

To measure displacements of a $3 \mathrm{D}$ reconstructed volume it is possible to resort to local or global DVC. Local DVC consists of correlating small volumes of interest $[41,42,43]$ as in 2D local DIC [29]. In the following a global and regularized scheme [40] will be considered. Gray level correlation residuals (see Equation (3)) will be used to detect damage. The sought displacement field will be decomposed onto a basis of continuous functions using 8-noded cubes with trilinear shape fonctions as proposed in classical finite element methods.

\subsection{Experimental procedure}

The X-ray microtomographic experiment is performed using the $\mathrm{X} 50+$ system (North Star Imaging, see Figure 12(a)) of LMT. A uniaxial specimen of the investigated SG cast iron is loaded in situ in a testing machine (Figure 12(b)). The custom made uniaxial device is placed on the turn table (while scanning the sample it is rotated $360^{\circ}$ along its vertical axis). The gripped specimen is placed inside the composite carbon tube whose purpose is to transmit the load and hold the load cell above the sample. The specimen 
is loaded from 200 up to $750 \mathrm{~N}$ in twelve incremental loading and unloading steps (Figure 13). To estimate the standard displacement resolution the first two scans were taken at equal load level $(F=200 \mathrm{~N})$.

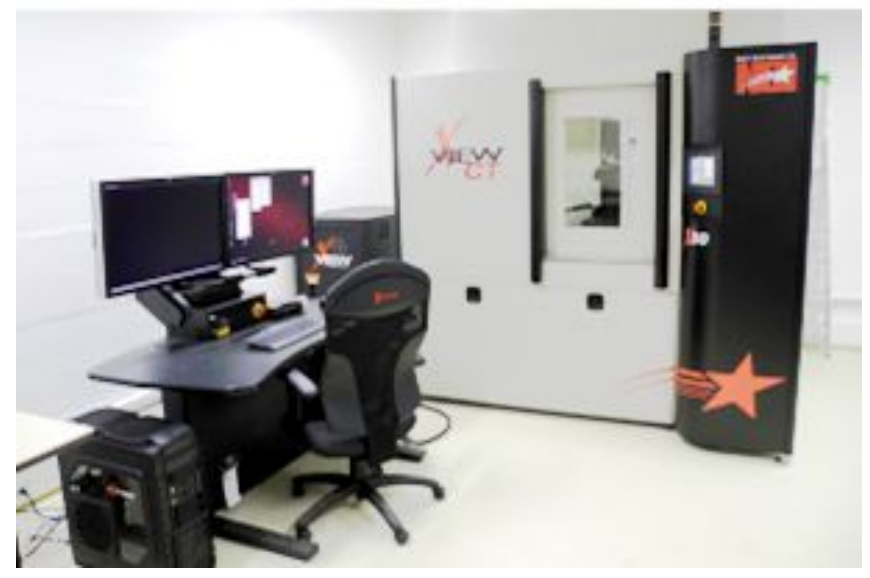

(a)

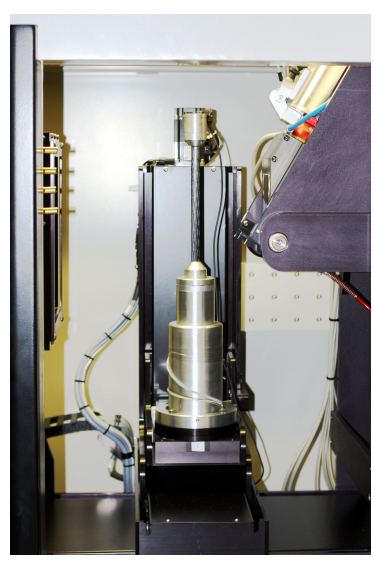

(b)

Figure 12: X50+ computed tomography system (a) and corresponding custom made uniaxial tensile machine for in situ experiments (b)

The dog-bone specimen is electrodischarge machined from a 1.6-mm thick plate. To ensure that the specimen will break in the ligament area and not in the grips the central part is thinned with a radius of $20 \mathrm{~mm}$ (Figure 14(a)). The ligament is $9-\mathrm{mm}$ high, while the smallest cross-section of the sample is $1.6 \times 1 \mathrm{~mm}^{2}$. Each tomographic scan was initially acquired with a definition of $1280 \times 1860$ pixels. The physical voxel size is $6.4 \mu \mathrm{m}$, and the reconstructed volume is encoded with 8-bit deep gray levels. In those 3D images, a region of interest is defined in which the displacement field will be measured. The size of the ROI was adjusted to the sample geometry (Figure 14(a)) focusing on the thinned part of the sample corresponding to an image size of $160 \times$ $196 \times 608$ voxels. 


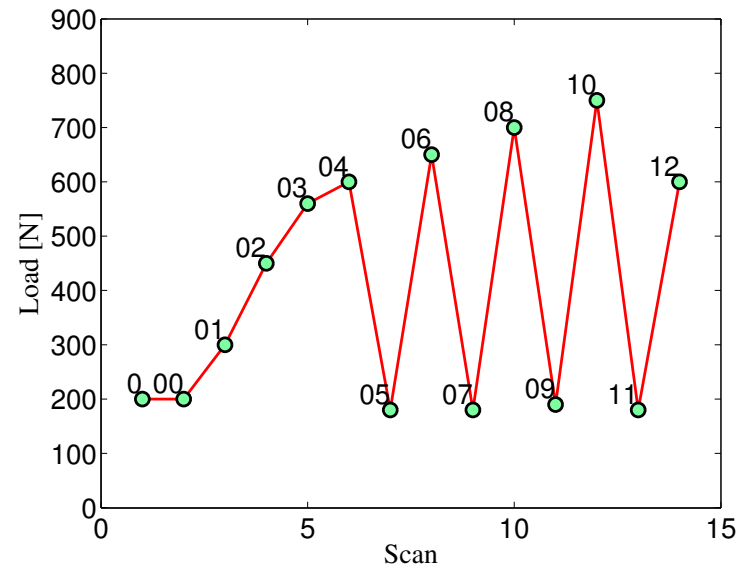

Figure 13: Loading history for the in situ experiment

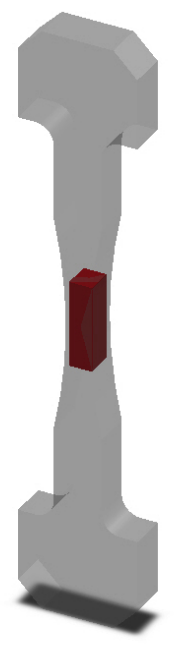

(a)

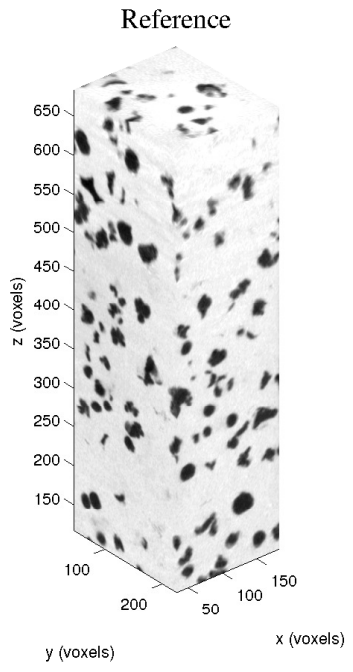

(b)

Figure 14: (a) Dog-bone sample used for tomography. The red zone inside the sample denotes the investigated ROI $(160 \times 196 \times 608$ voxels $)$. (b) Reference gray level ROI $(F=200 \mathrm{~N})$ 


\subsection{Uncertainty analysis}

Before studying the displacement field between two scans, it is important to evaluate the level of uncertainty attached to the natural texture of the image. Figure 14(b) shows the ROI of the reference scan used for DVC analyses. Two volumes acquired with the same load level $(F=200 \mathrm{~N})$ are correlated to evaluate the standard displacement resolution. Even though a fine contrast was found between the ferritic matrix and the graphite nodules, a large size of nodules is noted in comparison with previous studies [44, 45]. Since the investigated cast iron has a coarse microstructure it is necessary to make a compromise between the spatial and displacement resolutions. The only difficulty is to be able to capture the displacement without being trapped in secondary minima of the objective function. Two DVC algorithms (i.e., C8-DVC [39] and RC8-DVC [40]) are used to evaluate the measurement uncertainty.

First, C8-DVC was used. The smallest possible element size $\ell$ was 32 voxels. With smaller elements convergence was not reached. Bigger elements (i.e., 64 voxels) were also useless since the ROI width in the $x$ direction is 160 voxels. Regularized C8-DVC was also used with 16-voxel elements. The initial regularization length $\ell_{m}$ was chosen to be 64 voxels in the three

directions. During the relaxation process [46] $\ell_{m}$ is decreased by 16 -voxel increments in every new computation. When the regularization length was smaller than 16 voxels the algorithm could not converge since regularization has no significant impact if $\ell_{m}$ is less than the element size $\ell$.

Figure 15 shows the standard displacement resolution when C8 and RC8DVC are used to measure displacement fluctuations. When $\ell_{m}=64$ voxels is 
used a significant gap is noted when compared to classical C8-DVC. However, the relaxation of the regularization length increases the displacement resolution (i.e., the larger the regularization length, the smaller the displacement resolution). It is important to stress that the computation with 16-voxel elements converged even when $\ell_{m}=16$ voxels was used.

\subsection{Analysis of the results}

Several results are presented from RC8 analyses when $\ell_{m}=32$ voxels. The correlation was performed between the undeformed volume (scan 0) and deformed ones (scans 00-11). First, the displacement fields of the volumes corresponding to scan 05 (i.e., $560 \mathrm{~N}$ ) and the last loading scan 11 (i.e., 750 N) are studied, which show different distributions in the volume. Studying the displacement in the $x$-direction of scan 05 the contraction due to the Poisson's ratio is observed (Figure 16(a)). The displacement fields in $y$ and $z$-directions (Figure 16(c,e)) show that the sample was not loaded in pure tension. Additional rotations occur due to the gripping system of the testing machine. It is necessary to emphasize that no localization is observed on the displacement fields. However, the measured displacement fields for scan 11 show localized phenomena. Displacements in $x$ and $y$-directions (Figure 16(b,d)) show the presence of a macrocrack between $z=500$ and 600 voxels in the longitudinal direction. Virtually the same levels of standard displacement resolution (i.e., $\sigma_{x}=\sigma_{y}=\sigma_{z}=0.03$ voxel) were reported for the three directions in Figure 15. The measured displacement fluctuations reported for the two loading levels (Figure 16) are much higher when compared with the measurement resolution. It is concluded that the reported displacement fields are trustworthy. 


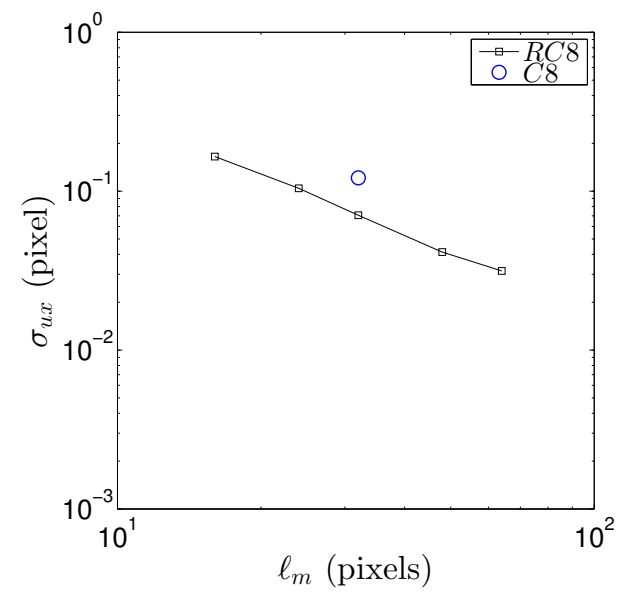

(a) $x$-direction

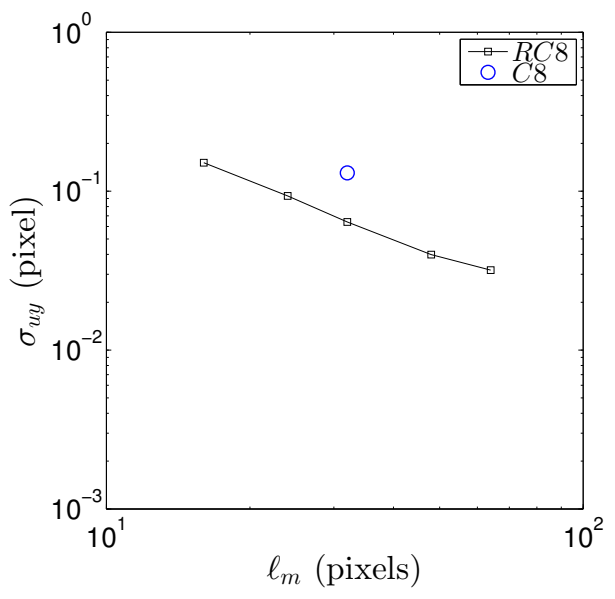

(b) $y$-direction

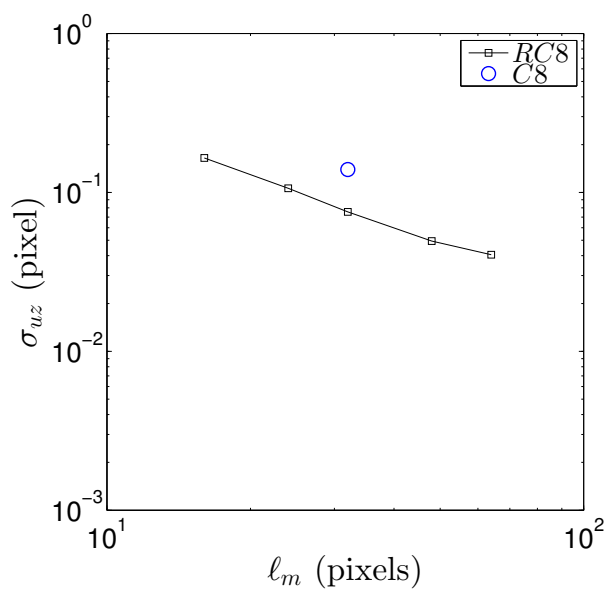

(c) $z$-direction

Figure 15: Standard displacement resolution with C8 and RC8-DVC for different regularization lengths $\ell_{m}$ (RC8-DVC) or element size $\ell$ (C8-DVC, circle). 16-voxel elements are used in RC8-DVC analyses 


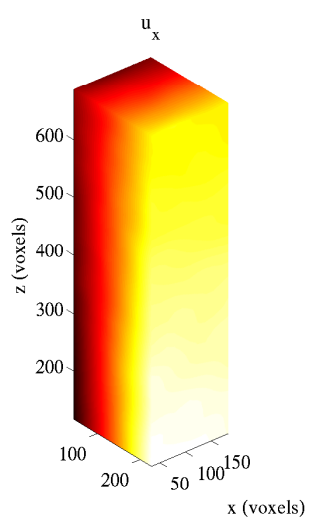

(a)

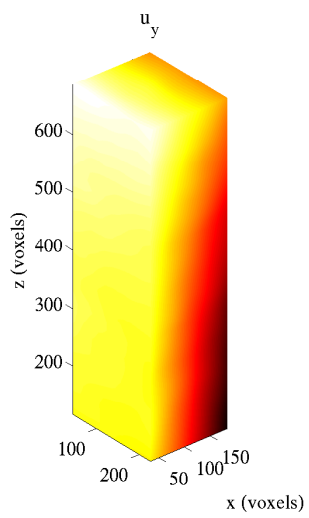

(c)

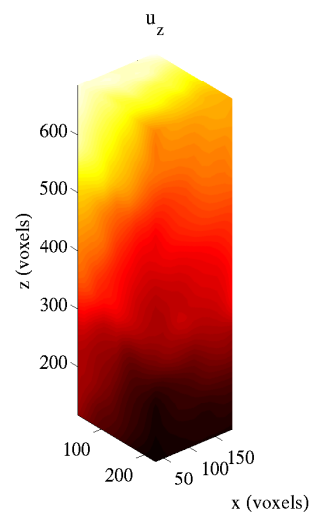

(e)

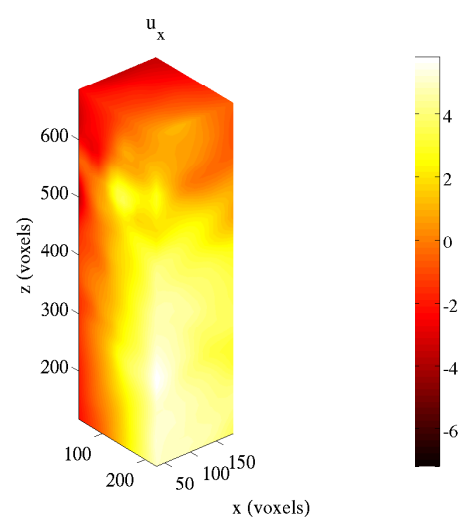

(b)

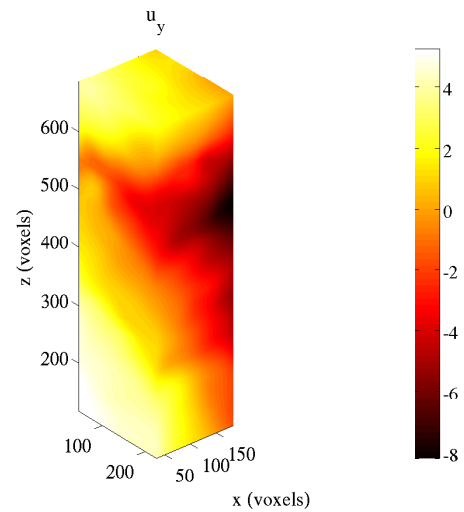

(d)

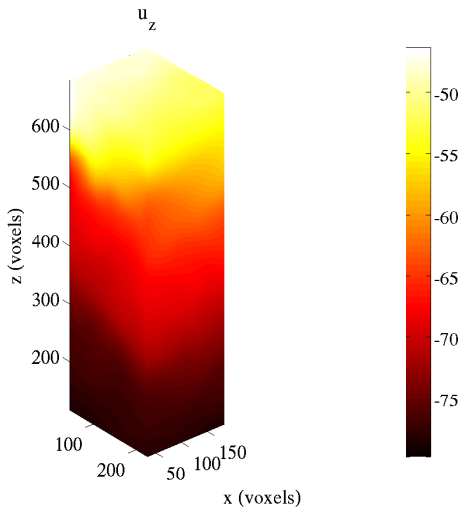

(f)

Figure 16: Measured displacement fields (exłressed in voxels, 1 voxel $\leftrightarrow 6.4 \mu \mathrm{m}$ ) in (a) \& (b) $x$, (b) \& (c) $y$ and (c) \& (d) z-directions corresponding to load levels $560 \mathrm{~N}$ (left) and $750 \mathrm{~N}$ (right) 
Von Mises' equivalent strain fields are shown in Figure 17(a,b). When the sample was loaded at $560 \mathrm{~N}$ (Figure 17(a)) two critical locations are detected, namely, one in the lower part (i.e., $z=200$ voxels) and another one in the upper part (i.e., $z=440$ voxels) of the ROI. For the load level corresponding to $760 \mathrm{~N}$ (Figure 17(b)) localization becomes dominant. Plotting the gray level residuals a damage mechanism is revealed in Figure 17(c,d). Observing the reference volume (Figure 14(b)) higher residual values are observed around the nodules. This phenomenon is caused by debonding between the matrix and the nodules. In the last step of loading the residuals show the macrocrack path that was caused by the localization of the displacement and strain fields.

In the sequel, the 12 volumes will be considered. The gray level residual maps (Figure 18) corresponding to the section normal to the $y$-axis $\varphi_{c}(x, y=$ 30 voxels, $z$ ) are chosen. The dynamic range of the residuals is selected so that an increase of fluctuations can be captured. Debonding between the matrix and the nodules is detected in the very early stages (i.e., even for scan 01). There is an increase of residuals as the loading levels become higher. Segmentation of the SG cast iron constituents even at the first loading level defines the damage micromechanism. This fact explains the loss of stiffness observed in the cyclic tensile experiment (see Figure 8).

From the SEM analysis of the broken sample (Figure 19) brittle damage is also recognized. Two brittle fracture mechanisms are found. First, transgranular damage is observed in the green window. Second, cleavage fracture is detected (in the red window of Figure 19). 


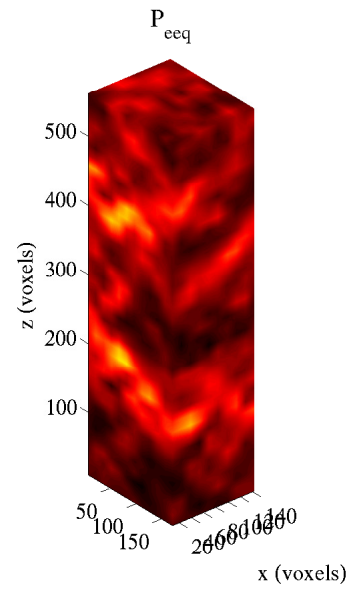

(a)

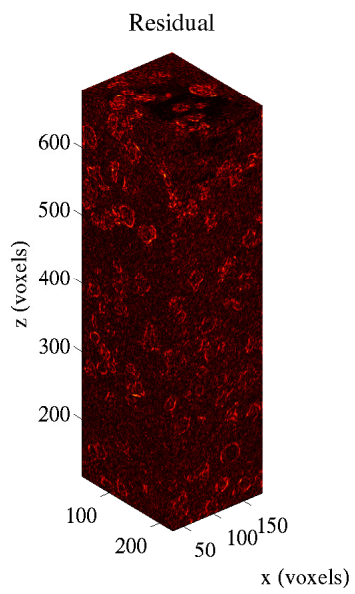

(c)

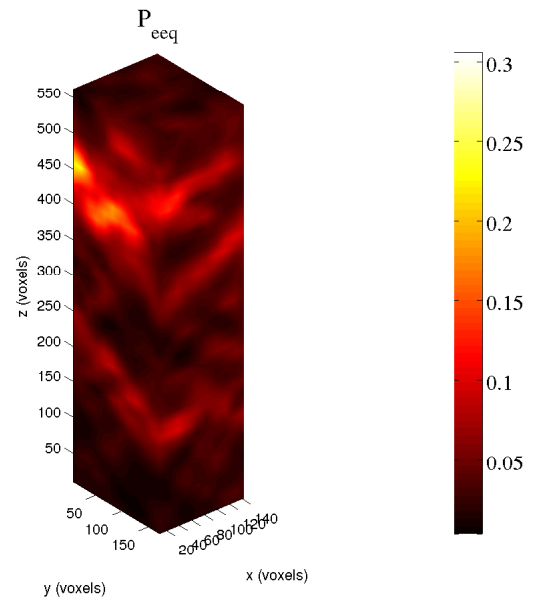

(b)

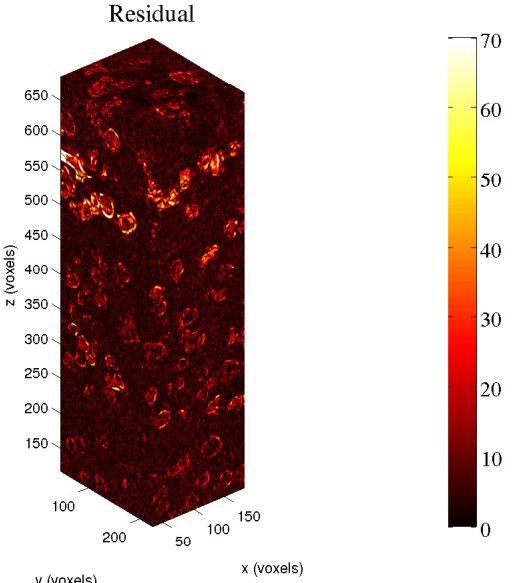

(d)

Figure 17: Von Mises' equivalent strain fields (a) \& (b), and gray level residual fields (c) $\&$ (d) for load levels $560 \mathrm{~N}$ (left) and $750 \mathrm{~N}$ (right) 


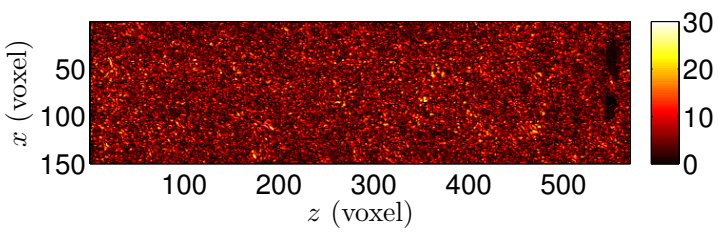

(a) scan00

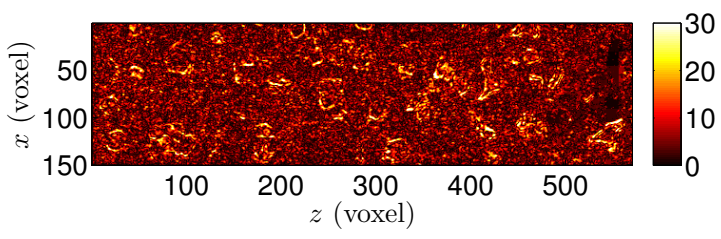

(c) scan02

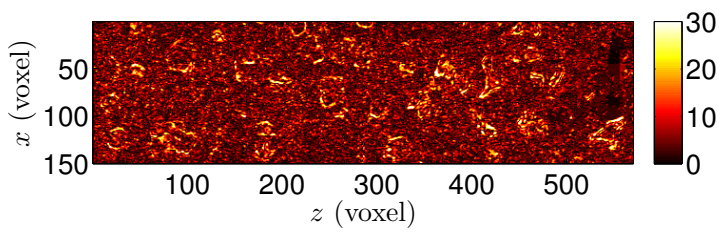

(e) scan04

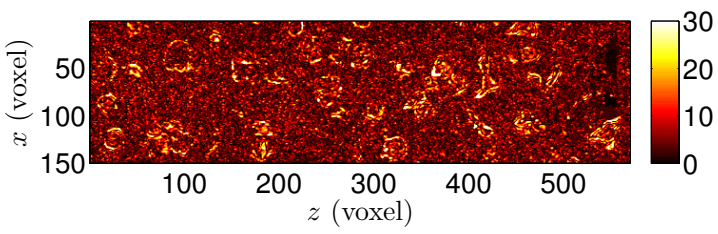

(g) scan06

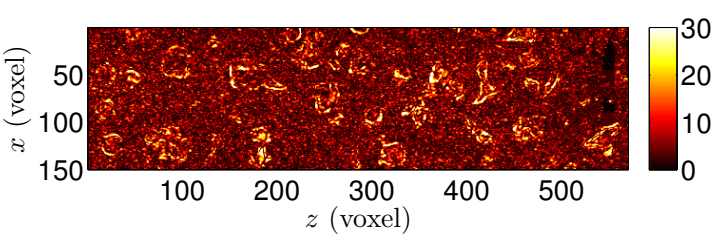

(i) $\operatorname{scan08}$

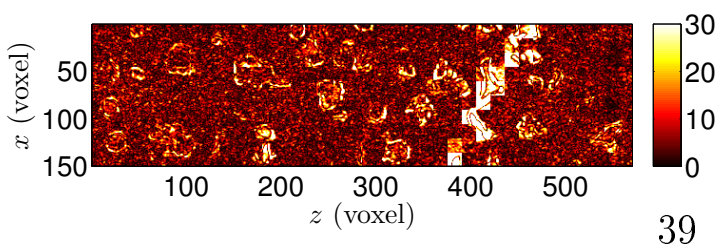

(k) scan 10

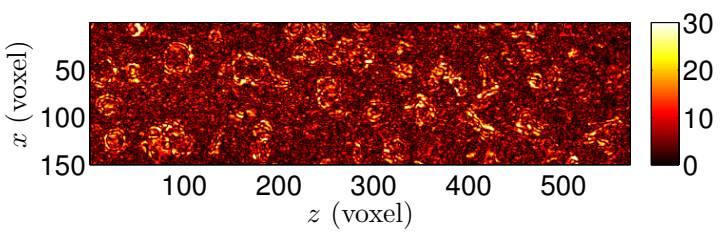

(b) scan01

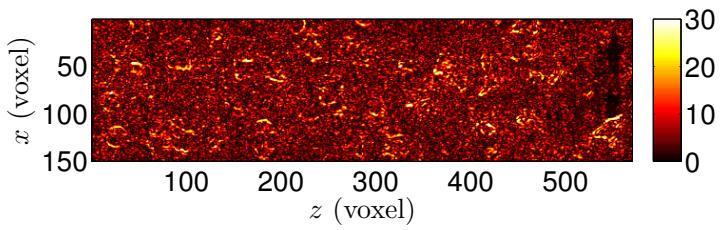

(d) $\operatorname{scan} 03$

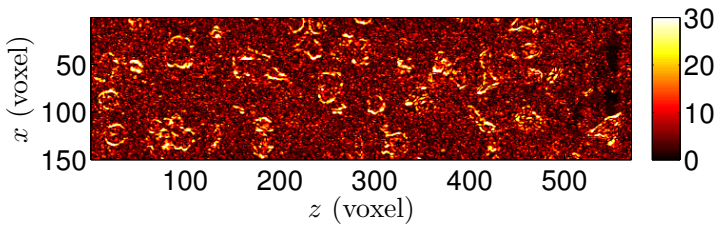

(f) scan05

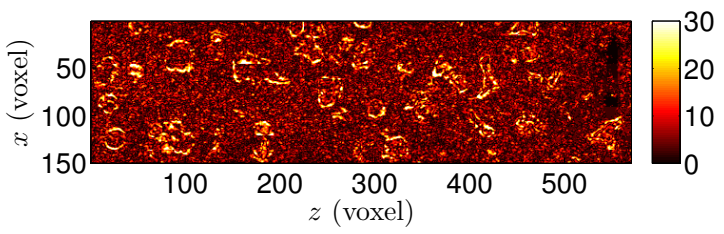

(h) scan07

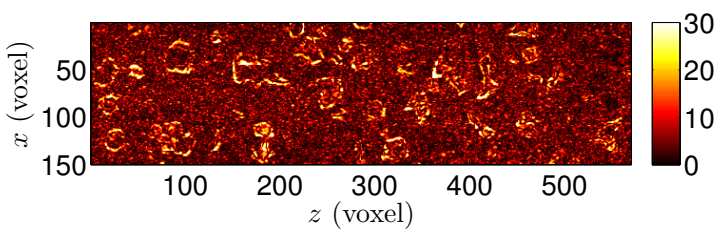

(j) scan09

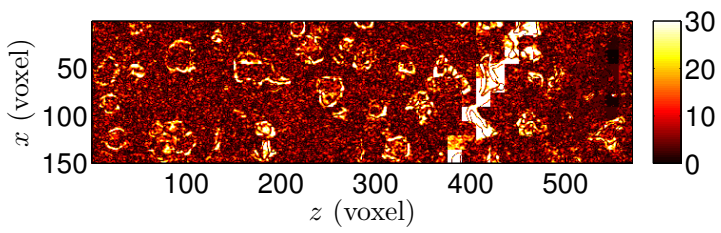

(1) scan 11

Figure 18: Gray level residual maps corresponding to the plane normal to the $y$-axis for the 12 scans 


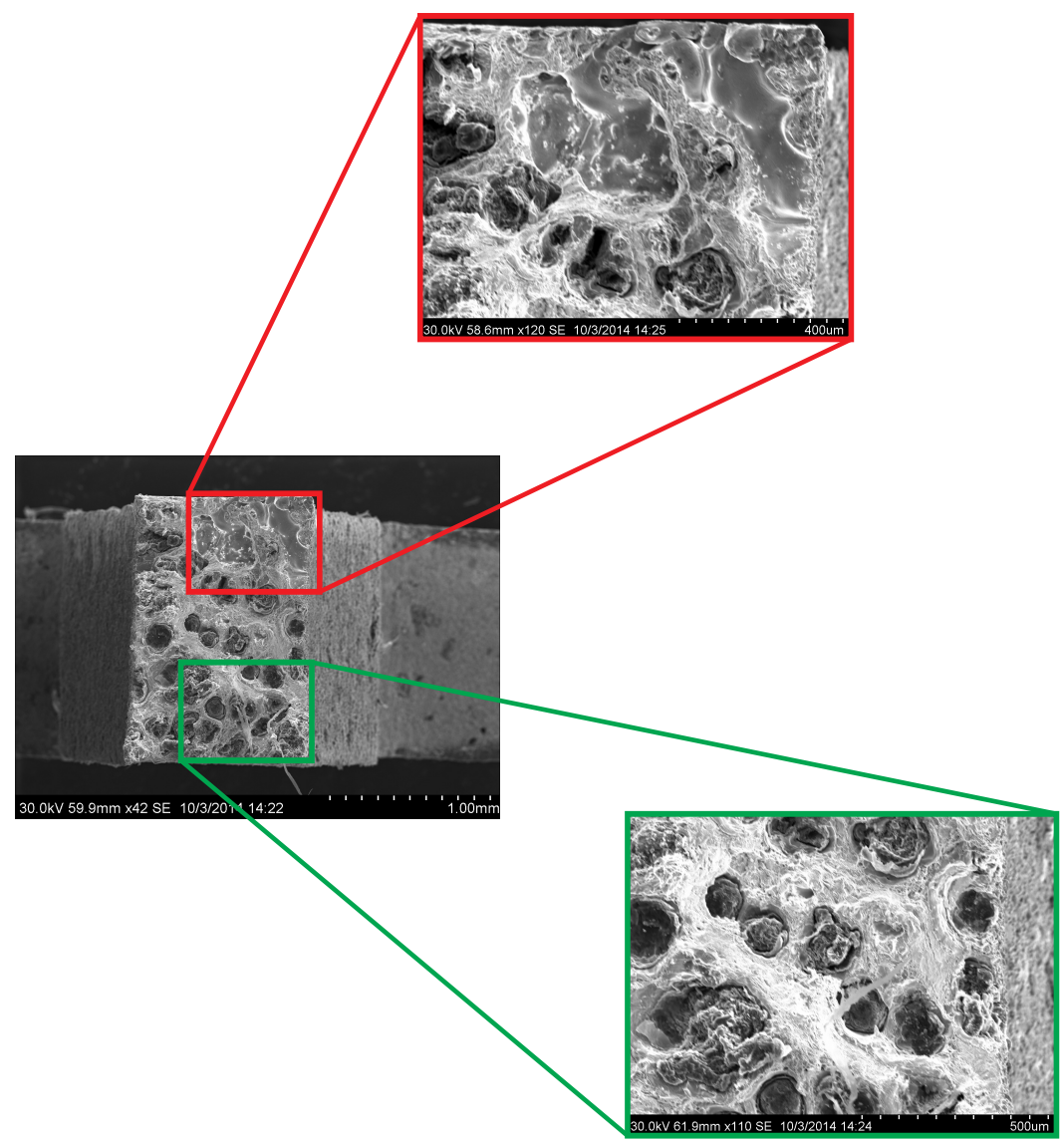

Figure 19: SEM analysis of the broken tomographic sample 


\section{Conclusions and perspectives}

The goal of this study was to identify elastic and nonlinear properties (i.e., isotropic and kinematic hardening) for a cyclic and uniaxial load history. The identification was performed via FEMU. Since global (FE-)DIC was used to measure displacement fields FE simulations were run in the same FE framework (i.e., the measured displacement field is obtained at the same nodes as the simulated displacement field [3]). The weighted FEMU-UF variant was used to minimize the total cost function comparing the measured and calculated displacement fields and static (load) information with sum of the reaction forces.

In the present work the two elastic parameters were first optimized in one identification procedure. The Poisson's ratio could be identified with FEMU-U and FEMU-UF variants [35]. However, the change of Young's modulus does not impact the displacement field in elasticity so only FEMUUF and FEMU-F could identify it. Consequently, the FEMU-UF variant is chosen. A good agreement was found for elastic parameters identified in a conventional way and via FEMU procedures.

Second, the identification of isotropic (Ludwik's law) and kinematic (Armstrong-Frederick's model) hardening postulates was performed. Initial (i.e., reference) input parameters were obtained with via standard curve fitting of the monotonic uniaxial response. The identified parameters with the proposed identification procedure yielded different residual levels for the cyclic loading history. When only the elastoplastic behavior is considered higher values of force residuals are reported. The reason why the cyclic loading regime cannot be described in a reliable way lies in modeling errors 
since Ludwik/Armstrong-Frederick's laws do not account for damage. When a coupled elastoplastic and damage law (i.e., Ludwik's isotropic hardening and Lemaitre's damage model) is considered the force residuals are the lowest. The latter is the best of the three models in the present case.

By measuring the displacement fields at a macroscale it was detected that the investigated SG cast iron has a low elongation to failure. When identifying the elastoplastic material response early damage initiation was

observed. From the latter the micromechanism could not be distinguished. Hence, a cyclic experiment was carried out in an X-ray tomograph. The correlation residuals revealed that debonding between the matrix and the nodules occurs at the first analyzed loading step. It is worth noting that this experiment was used herein to reveal the damage micromechanisms. It may also be used to identify or validate plastic and damage models at the microscale.

The next part of the present study will consist of assessing elastoplastic parameters from biaxial experiments where three different loading histories have been applied to the same material [47]. The parameters determined herein will therefore be initial guesses for the same type of updating procedure. It will therefore be possible to probe the predictive capacity of the elastoplastic models studied herein for more complex loadings and specimen geometry.

\section{Acknowledgments}

ZT thanks Campus France that supported his stay at LMT through an Eiffel scholarship. This work has benefited from the support of the French 
'Agence Nationale de la Recherche' through the 'Investissements d'Avenir' program under the reference ANR-10-EQPX-37 MATMECA. The experiment conducted on X-ray tomograph was performed with the help of Dr. Amine Bouterf. The authors would also like to warmly thank Dr. Stéphane Roux for numerous discussions on issues introduced in the present paper.

\section{References}

[1] Grédiac, M., and Hild, F. Full-Field Measurements and Identification in Solid Mechanics. Wiley-ISTE 2012.

[2] Avril, S., Bonnet, M., Bretelle, A.-S., Grédiac, M., Hild, F., Ienny, P., Latourte, F., Lemosse, D., Pagano, S., Pagnacco, E., and Pierron, F. Overview of identification methods of mechanical parameters based on full-field measurements. Experimental Mechanics 2008; 48(4):381-402.

[3] Gras, R., Leclerc, H., Roux, S., Otin, S., Schneider, J., and Périé, J.-N. Identification of the out-of-plane shear modulus of a 3D woven composite. Experimental Mechanics 2013; 53(5):719-730.

[4] Grédiac, M., and Pierron, F. Applying the virtual fields method to the identification of elasto-plastic constitutive parameters. International Journal of Plasticity 2006; 22(4):602-627.

[5] Cooreman, S., Lecompte, D., Sol, H., Vantomme, J., and Debruyne, D. Elasto-plastic material parameter identification by inverse methods: Calculation of the sensitivity matrix. International Journal of Solids and Structures 2007; 44(13):4329-4341. 
[6] Robert, L., Velay, V., Decultot, N., and Ramde, S. Identification of hardening parameters using finite element models and full-field measurements: some case studies. Journal of Strain Analysis for Engineering Design 2012; 47(1):3-17.

[7] Réthore, J., Muhibullah, Elguedj, T., Coret, M., Chaudet, P., and Combescure, A. Robust identification of elasto-plastic constitutive law parameters from digital images using 3D kinematics. International Journal of Solids and Structures 2013; 50(1):73-85.

[8] Mathieu, F., Leclerc, H., Hild, F., and Roux, S. Estimation of elastoplastic parameters via weighted FEMU and integrated-DIC. Experimental Mechanics 2015; 55(1):105-119.

[9] Claire, D., Hild, F., and Roux, S. Identification of damage fields using kinematic measurements. Comptes-Rendus Mecanique 2002; 330:729734 .

[10] Chalal, H., Meraghni, F., Pierron, F., and Grédiac, M. Direct identification of the damage behaviour of composite materials using the virtual fields method. Composites: Part A 2004; 35:841-848.

[11] Réthoré, J. A fully integrated noise robust strategy for the identification of constitutive laws from digital images. International Journal for Numerical Methods in Engineering 2010; 84(6):631-660.

[12] Wu, T., Coret, M., and Combescure, A. Strain Localisation and Damage Measurement by Full 3D Digital Image Correlation: Application to 15 5PH Stainless Steel. Strain 2011; 47(1): 49-61. 
[13] Bouterf, A., Roux, S., Hild, F., G. Vivier, X. Brajer, Maire, E., and Meille, S. Damage law identification from full field displacement measurement: Application to four-point bending test for plasterboard. European Journal of Mechanics A/Solids 2015; 49:60-66.

[14] Grédiac, M. Principe des travaux virtuels et identification. Comptes Rendus de l'Académie des Sciences (in French with abridged English version) 1989; II(309):1-5.

[15] Claire, D., Hild, F., and Roux, S. A finite element formulation to identify damage fields: the equilibrium gap method. International Journal for Numerical Methods in Engineering 2004; 61(2):189-208.

[16] Claire, D., Hild, F., and Roux, S. Identification of a damage law by using full-field displacement measurements. International Journal of Damage Mechanics 2007; 16(2):179-197.

[17] Avril, S., Pierron, F., Sutton, M. A., and Yan, J. Identification of elasto-visco-plastic parameters and characterization of luders behavior using digital image correlation and the virtual fields method. Mechanics of Materials 2008; 40(9):729-742.

[18] Pierron, F., Avril, S., and Tran, V. Extension of the virtual fields method to elasto-plastic material identification with cyclic loads and kinematic hardening. International Journal of Solids and Structures 2010; 47(2223):2993-3010.

[19] Latourte, F., Chrysochoos, A., Pagano, S., and Wattrisse, D. Elasto- 
plastic behavior identification for heterogeneous loadings and materials. Experimental Mechanics 2008; 48(4):435-449.

[20] Hild, F., and Roux, S. Digital image correlation: from displacement measurement to identification of elastic properties - a review. Strain 2006; 42:69-80.

[21] Roux, S. and Hild, F. Stress intensity factor measurements from digital image correlation: post-processing and integrated approaches. International Journal of Fracture 2006; 140(1/4):141-157.

[22] Leclerc H, Périé J-N, Roux S, Hild F. Integrated digital image correlation for the identification of mechanical properties. MIRAGE 2009, Gagalowicz A, Philips W, eds. Springer: Berlin, 2009; 161-171.

[23] Roux, S. and Hild, F. Digital image mechanical identification (DIMI). Experimental Mechanics 2008; 48(4):495-508.

[24] Rossi, M., Broggiato, G., and Papalini, S. Identification of ductile damage parameters using digital image processing. SEM Annual Conference and Exposition on Experimental and Applied Mechanics - Experimental Mechanics 2006.

[25] Broggiato, G. Adaptive image correlation technique for full-field strain measurement. Proceedings 12th Int. Conf. Exp. Mech. 2004.

[26] Sun, Y., Pang, J., Wong, C., and Su, F. Finite element formulation for a digital image correlation method. Applied Optics 2005; 43(34):73577363. 
[27] Besnard, G., Hild, F., and Roux, S. "finite-element" displacement fields analysis from digital images: Application to Portevin-Le Chatelier bands. Experimental Mechanics 2006; 46(6):789-804.

[28] Hild, F. and Roux, S. Comparison of local and global approaches to digital image correlation. Experimental Mechanics 2012; 52(9):15031519 .

[29] Sutton, M., Wolters, W., Peters, W., Ranson, W., and McNeill, S. Determination of displacements using an improved digital correlation method. Image and Vision Computing 1983; 1(3):133 - 139.

[30] Chu, T., Ranson, W., and Sutton, M. Applications of digital-imagecorrelation techniques to experimental mechanics. Experimental Mechanics 1985; 25(3):232-244.

[31] Schreier, H. and Sutton, M. Systematic errors in digital image correlation due to undermatched subset shape functions. Experimental $\mathrm{Me}$ chanics 2002; 42(3):303-310.

[32] Ludwik, P. Elemente der technologischen Mechanik. Verlag von Julius Springer 1909; Berlin.

[33] Armstrong, P. and Frederick, C. A mathematical representation of the multiaxial bauschinger effect. Report $R D / B / N$ 731, Central Electricity Generating Board 1966.

[34] Prager, W. A new method of analysing stress and strain in work hardening plastic solids. ASME Journal of Applied Mechanics 1956; 78(493). 
[35] Tomičević, Z. Identification of the mechanical properties of nodular graphite cast iron via multiaxial tests. PhD thesis of ENS Cachan and the University of Zagreb 2015.

[36] Lemaitre, J. How to use damage mechanics. Nucl. Eng. Design 1984; 80:233-245.

[37] Bertin, M., Hild, F., Roux, S., Mathieu, F., Leclerc, H., Aimedieu, P. Integrated Digital Image Correlation applied to elasto-plastic identification in a biaxial experiment. J. Strain Analysis 2016; 51(2):118-131.

[38] Lemaitre, J. and Desmorat, R. Engineering Damage Mechanics:Ductile, Creep, Fatigue and Brittle Failures 2005. Springer.

[39] Roux, S., Hild, F., Viot, P., and Bernard, D. Three-dimensional image correlation from X-Ray computed tomography of solid foam. Composites Part A: Applied Science and Manufacturing 2008; 39(8):1253-1265.

[40] Taillandier-Thomas, T., Roux, S., Morgeneyer, T., and Hild, F. Localized strain field measurement on laminography data with mechanical regularization. Nuclear Instruments and Methods in Physics Research Section B: Beam Interactions with Materials and Atoms 2014; 324(0):70-79.

[41] Bay, B., Smith, T., Fyhrie, D., and Saad, M. Digital volume correlation: Three-dimensional strain mapping using x-ray tomography. Experimental Mechanics 1999; 39(3):217-226. 
[42] Smith, T., Bay, B., and Rashid, M. Digital volume correlation including rotational degrees of freedom during minimization. Experimental Mechanics 2002; 42(3):272-278.

[43] Verhulp, E., van Rietbergen, B., and Huiskes, R. A three-dimensional digital image correlation technique for strain measurements in microstructures. Journal of Biomechanics 2004; 37(9):1313-1320.

[44] Limodin, N., Réthoré, Y., Buffière, J.-Y., Gravouil, A., Hild, F., and Roux, S. Crack closure and stress intensity factor measurements in nodular graphite cast iron using three-dimensional correlation of laboratory x-ray microtomography images. Acta Materialia 2009; 57:4090-4101.

[45] Limodin, N., Réthoré, Y., Buffière, J.-Y., Hild, F., Roux, S., Ludwig, W., Rannou, J., and Gravouil, A. Influence of closure on the 3d propagation of fatigue cracks in a nodular cast iron investigated by x-ray tomography and 3d volume correlation. Acta Materialia 2010; 58(8):2957 $-2967$.

[46] Tomičević, Z., Hild, F., and Roux, S. Mechanics-aided digital image correlation. Journal of Strain Analysis for Engineering Design 2013; 48(5):330-343.

[47] Tomičević, Z., Kodvanj, J., Hild, F. Characterization of the nonlinear behavior of nodular graphite cast iron via inverse identification - Analysis of biaxial tests. Submitted for publication 2015. 


\section{List of Figures}

1 Metallography of the investigated SG cast iron . . . . . . . 5

2 Loading history for the uniaxial cyclic experiment. The image acquisition rate was 1 frame $/ \mathrm{s} \ldots \ldots . \ldots 7$

3 Reference picture of the uniaxial experiment used for a DIC analysis with 25-pixel elements. The blue rectangle depicts the zone where the DIC gauge was measuring the mean strain. In the FEMU procedure, boundary conditions measured with DIC were prescribed on the edge nodes depicted with green

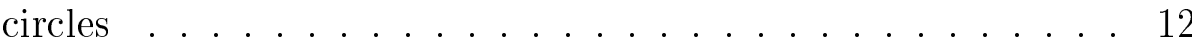

4 Measured displacement fields via T3-DIC in (a) transverse and (b) longitudinal directions for a load level corresponding to $18 \mathrm{kN}$. The displacements are expressed in pixels (1 pixel $\leftrightarrow$ $52 \mu \mathrm{m}) \ldots \ldots \ldots \ldots \ldots \ldots \ldots$

5 (a) Comparison of measured force and sum of reaction forces computed with initial and identified material parameters for Ludwik's law. (b) Difference between measured and predicted load levels for the initial and optimized elastic parameters . . 19

6 (a) Comparison of measured force and sum of reaction forces computed with the initial and identified parameters for Armstrong-Frederick's constitutive law. (b) Difference between measured and predicted load levels for the initial and optimized parameters ................ 20

7 (a) Strain history in longitudinal (i.e., axial) and transverse directions. (b) Stress/strain curve for the cyclic uniaxial test . 22 
8 Change of Young's modulus with cumulative plastic strain . . 23

9 (a) Comparison of the measured load and sum of reaction forces computed with the initial and identified material parameters of an elastoplastic law coupled with damage for one element. (b) Difference between measured and predicted load levels for the initial and optimized parameters . . . . . . . 25

10 (a) Comparison of measured force and sum of reaction forces computed with the initial and identified parameters of the elastoplastic and damage law. (b) Difference between measured and predicted load levels for the initial and optimized

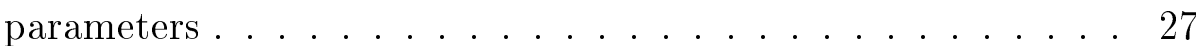

11 Comparison between the uniaxial stress/strain response of the three identified models and the reference obtained with strain gauge and load cell data. (b) Difference between measured and predicted stresses by the three studied models . . . . . 29

$12 \mathrm{X} 50+$ computed tomography system (a) and corresponding custom made uniaxial tensile machine for in situ experiments

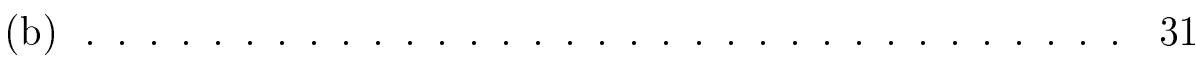

13 Loading history for the in situ experiment . . . . . . . . . 32

14 (a) Dog-bone sample used for tomography. The red zone inside the sample denotes the investigated ROI $(160 \times 196 \times 608$ voxels). (b) Reference gray level ROI $(F=200 \mathrm{~N})$. . . . . . 32 
15 Standard displacement resolution with C8 and RC8-DVC for different regularization lengths $\ell_{m}$ (RC8-DVC) or element size $\ell$ (C8-DVC, circle). 16-voxel elements are used in RC8-DVC

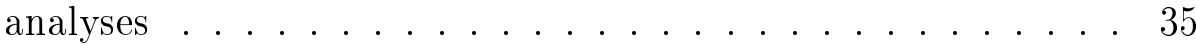

16 Measured displacement fields (expressed in voxels, 1 voxel $\leftrightarrow$ $6.4 \mu \mathrm{m})$ in (a) \& (b) $x$, (b) \& (c) $y$ and (c) \& (d) z-directions corresponding to load levels $560 \mathrm{~N}$ (left) and $750 \mathrm{~N}$ (right) . . 36

17 Von Mises' equivalent strain fields (a) \& (b), and gray level residual fields (c) \& (d) for load levels $560 \mathrm{~N}$ (left) and $750 \mathrm{~N}$

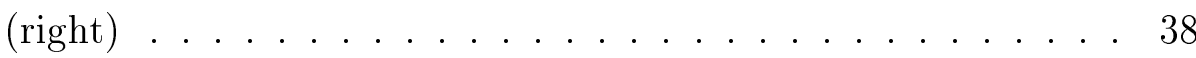

18 Gray level residual maps corresponding to the plane normal to the $y$-axis for the 12 scans . . . . . . . . . . . 39

19 SEM analysis of the broken tomographic sample . . . . . . . 40 


\section{List of Tables}

1 Initial and identified elastic parameters for cyclic uniaxial tension 16

2 Identified Ludwik's parameters via FEMU-UF . . . . . . . . . 18

3 Identified Armstrong-Frederick's parameters with the FEMU-

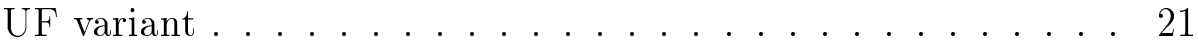

4 Identified elastoplastic and damage parameters on one element via FEMU-F . . . . . . . . . . . . . . . . . 24

5 Identified elastoplastic and damage parameters with an FE

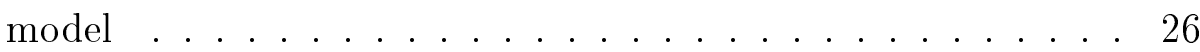

6 Identification residuals for the three analyzed models . . . . 28 